\title{
The Pascal Triangle of a Discrete Image: Definition, Properties and Application to Shape Analysis ${ }^{\star}$
}

\author{
Mireille BOUTIN ${ }^{\dagger}$ and Shanshan $H U A N G \ddagger$ \\ $\dagger$ School of Electrical and Computer Engineering, Purdue University, USA \\ E-mail: mboutin@purdue.edu \\ $\ddagger$ Department of Mathematics, Purdue University, USA \\ E-mail: huang94@purdue.edu
}

Received September 24, 2012, in final form April 03, 2013; Published online April 11, 2013

http://dx.doi.org/10.3842/SIGMA.2013.031

\begin{abstract}
We define the Pascal triangle of a discrete (gray scale) image as a pyramidal arrangement of complex-valued moments and we explore its geometric significance. In particular, we show that the entries of row $k$ of this triangle correspond to the Fourier series coefficients of the moment of order $k$ of the Radon transform of the image. Group actions on the plane can be naturally prolonged onto the entries of the Pascal triangle. We study the prolongation of some common group actions, such as rotations and reflections, and we propose simple tests for detecting equivalences and self-equivalences under these group actions. The motivating application of this work is the problem of characterizing the geometry of objects on images, for example by detecting approximate symmetries.
\end{abstract}

Key words: moments; symmetry detection; moving frame; shape recognition

2010 Mathematics Subject Classification: 30E05; 57S25; 68T10

\section{Definition and reconstruction properties}

Let $\left\{\left(x_{k}, y_{k}\right)\right\}_{k=1}^{N}$ with $x_{k}, y_{k} \in \mathbb{R}$ represent the pixel locations of a digital image. For simplicity, we use complex coordinates $z_{k}=x_{k}+i y_{k}$. Consider a gray scale image defined on $\left\{z_{k}\right\}_{k=1}^{N}$. More specifically, we have a mapping $\rho:\left\{z_{k}\right\}_{k=1}^{N} \rightarrow \mathbb{R}_{\geq 0}$, where $\rho\left(z_{k}\right)$ represents the intensity of pixel $z_{k} \cdot{ }^{1}$ Denote the discrete gray scale image by $I=\left\{\left(z_{k}, \rho\left(z_{k}\right)\right)\right\}_{k=1}^{N}$.

Consider the following moment matrix:

$$
\tau_{N}(I)=\left(\begin{array}{cccccc}
\mu_{0,0} & \mu_{1,0} & \mu_{2,0} & \mu_{3,0} & \cdots & \mu_{N-1,0} \\
\mu_{0,1} & \mu_{1,1} & \mu_{2,1} & \cdots & \cdots & \mu_{N-1,1} \\
\mu_{0,2} & \mu_{1,2} & \cdots & \cdots & \cdots & \mu_{N-1,2} \\
\mu_{0,3} & \cdots & \cdots & \cdots & \cdots & \mu_{N-1,3} \\
\vdots & & & & & \vdots \\
\mu_{0, N-1} & \mu_{1, N-1} & \cdots & \cdots & \cdots & \mu_{N-1, N-1}
\end{array}\right)_{N \times N}
$$

where $\mu_{j, l}=\sum_{k=1}^{N} z_{k}^{j} \bar{z}_{k}^{l} \rho\left(z_{k}\right), j, l \in \mathbb{Z}_{\geq 0}$ is the complex moment of order $(j, l)$ for the discrete image $I$. Observe the conjugate symmetry property of the moments $\mu_{j, l}=\bar{\mu}_{l, j}$. In particular, $\mu_{j, j} \in \mathbb{R}, \forall j \in \mathbb{Z}_{\geq 0}$.

${ }^{\star}$ This paper is a contribution to the Special Issue "Symmetries of Differential Equations: Frames, Invariants and Applications". The full collection is available at http://www.emis.de/journals/SIGMA/SDE2012.html

${ }^{1}$ We use $\mathbb{R}_{\geq 0}$ instead of a specific discrete domain such as $\{0,1, \ldots, 255\}$ for more generality. 
We can express the relationship between the moments and the image $I$ in matrix form:

$$
\tau_{N}(I)=Z^{\dagger} W Z
$$

where

$$
Z=\left(\begin{array}{ccccc}
1 & z_{1} & z_{1}^{2} & \cdots & z_{1}^{N-1} \\
1 & z_{2} & z_{2}^{2} & \cdots & z_{2}^{N-1} \\
\vdots & \vdots & \cdots & \vdots & \\
1 & z_{N} & z_{N}^{2} & \cdots & z_{N}^{N-1}
\end{array}\right), \quad W=\left(\begin{array}{cccc}
\rho\left(z_{1}\right) & 0 & \cdots & 0 \\
0 & \rho\left(z_{2}\right) & \cdots & 0 \\
\vdots & \vdots & \ddots & \vdots \\
0 & \cdots & 0 & \rho\left(z_{N}\right)
\end{array}\right)
$$

and $Z^{\dagger}$ is the conjugate transpose of $Z$. Observe that $Z$ is a Vandermonde matrix and therefore is invertible when the pixel locations $z_{k}$ are pairwise distinct. Therefore, if the pixel coordinates are known and pairwise distinct, one can reconstruct the image $I$ by matrix inversion: $W=$ $\left(Z^{-1}\right)^{\dagger} \tau_{N}(I) Z^{-1}$.

Definition 1. Let $r$ be a nonnegative integer and let $I$ be a discrete gray scale image. The Pascal triangle $T^{r}(I)$ of order $r$ of $I$ is the following pyramid:

$$
\begin{aligned}
& \mu_{0,0} \\
& \mu_{0,1} \quad \mu_{1,0} \\
& \mu_{0,2} \quad 2 \mu_{1,1} \quad \mu_{2,0} \\
& \mu_{0,4} \quad \mu_{0,3} \quad 3 \mu_{1,2} \quad \mu_{1,3} \quad 3 \mu_{2,1} \mu_{2,2} \mu_{3,0} \quad \mu_{3,1} \quad \mu_{4,0} \\
& \begin{array}{ccccc} 
& & \vdots & & \\
\mu_{0, r}\left(\begin{array}{c}
r \\
1
\end{array}\right) \mu_{1, r-1} & \ldots & \left(\begin{array}{c}
r \\
l
\end{array}\right) \mu_{l, r-l} & \ldots & \left(\begin{array}{c}
r \\
r-1
\end{array}\right) \mu_{r-1,1} \mu_{r, 0}
\end{array}
\end{aligned}
$$

Lemma 1 (pixel intensity reconstruction property). If the grid point locations $\left\{z_{k}\right\}_{k=1}^{N}$ are known and pairwise distinct, then the image $I$ can be reconstructed from the Pascal triangle $T^{N-1}(I)$ of order $N-1$. More specifically, knowledge of the entries of the right diagonal row of $T^{N-1}(I)$, i.e. $\left\{\mu_{j, 0}\right\}_{j=0}^{N-1}$, is sufficient for image reconstruction ${ }^{2}$.

Proof. Recall the definition of the moments

$$
\mu_{j, l}=\sum_{k=1}^{N} z_{k}^{j} \bar{z}_{k}^{l} \rho\left(z_{k}\right) .
$$

We consider the vector formed by the moments $\left\{\mu_{j, 0}\right\}_{j=0}^{N-1}$, which can be written in matrix form as

$$
\left(\begin{array}{c}
\mu_{0,0} \\
\mu_{1,0} \\
\mu_{2,0} \\
\vdots \\
\mu_{N-1,0}
\end{array}\right)=\left(\begin{array}{cccc}
1 & 1 & \cdots & 1 \\
z_{1} & z_{2} & \cdots & z_{N} \\
z_{1}^{2} & z_{2}^{2} & \cdots & z_{N}^{2} \\
\vdots & \vdots & \vdots & \vdots \\
z_{1}^{N-1} & z_{2}^{N-1} & \cdots & z_{N}^{N-1}
\end{array}\right)\left(\begin{array}{c}
\rho\left(z_{1}\right) \\
\rho\left(z_{2}\right) \\
\vdots \\
\rho\left(z_{N}\right)
\end{array}\right) .
$$

Observe that the coefficient matrix in (2) is a Vandermonde matrix. The Vandermonde matrix has full rank when $z_{j} \neq z_{k}$ for all distinct $j, k=1,2, \ldots, N$. Thus, since the pixel locations are assumed to be distinct, we can reconstruct the pixel intensities $\left\{\rho\left(z_{k}\right)\right\}_{k=1}^{N}$ by inverting the coefficient matrix and multiplying by the moment vector on the left-hand-side.

\footnotetext{
${ }^{2}$ The fact that the pixel intensities can be reconstructed from a finite number of moments was stated in [3]. Our lemma provides a clear statement of the conditions under which this reconstruction is theoretically possible.
} 
Notice that if we consider the Pascal triangle $T^{N}(I)$ of order $N$, then knowledge of the second right diagonal row of $T^{N}(I)$, i.e. $\left\{\mu_{j, 1}\right\}_{j=0}^{N-1}$, is also sufficient for image reconstruction as long as the $z_{k}$ 's are pairwise distinct and nonzero. This is because the vector formed by the moments $\left\{\mu_{j, 1}\right\}_{j=0}^{N-1}$ can be written in matrix form as

$$
\begin{aligned}
\left(\begin{array}{c}
\mu_{0,1} \\
\mu_{1,1} \\
\mu_{2,1} \\
\vdots \\
\mu_{N-1,1}
\end{array}\right)= & \left(\begin{array}{cccc}
\bar{z}_{1} & \bar{z}_{2} & \cdots & \bar{z}_{N} \\
z_{1} \bar{z}_{1} & z_{2} \bar{z}_{2} & \cdots & z_{N} \bar{z}_{N} \\
z_{1}^{2} \bar{z}_{1} & z_{2}^{2} \bar{z}_{2} & \cdots & z_{N}^{2} \bar{z}_{N} \\
\vdots & \vdots & \vdots & \vdots \\
z_{1}^{N-1} \bar{z}_{1} & z_{2}^{N-1} \bar{z}_{2} & \cdots & z_{N}^{N-1} \bar{z}_{N}
\end{array}\right)\left(\begin{array}{c}
\rho\left(z_{1}\right) \\
\rho\left(z_{2}\right) \\
\vdots \\
\rho\left(z_{N}\right)
\end{array}\right) \\
& \left(\begin{array}{cccc}
1 & 1 & \cdots & 1 \\
z_{1} & z_{2} & \cdots & z_{N} \\
z_{1}^{2} & z_{2}^{2} & \cdots & z_{N}^{2} \\
\vdots & \vdots & \vdots & \vdots \\
z_{1}^{N-1} & z_{2}^{N-1} & \cdots & z_{N}^{N-1}
\end{array}\right)\left(\begin{array}{cccc}
\bar{z}_{1} & 0 & \cdots & 0 \\
0 & \bar{z}_{2} & \cdots & 0 \\
\vdots & \vdots & \ddots & \vdots \\
0 & 0 & \cdots & \bar{z}_{N}
\end{array}\right)\left(\begin{array}{c}
\rho\left(z_{1}\right) \\
\rho\left(z_{2}\right) \\
\vdots \\
\rho\left(z_{N}\right)
\end{array}\right)
\end{aligned}
$$

The coefficient matrix in (3) is a Vandermonde matrix multiplied by a diagonal matrix. Assuming that the pixel locations are pairwise distinct insures that the Vandermonde matrix is invertible, and further assuming that they are nonzero insures invertibility of the diagonal matrix. Hence the coefficient matrix in (3) is nonsingular and we can reconstruct the pixel intensities $\left\{\rho\left(z_{k}\right)\right\}_{k=1}^{N}$ from $T^{N}(I)$ by inverting this coefficient matrix and multiplying by the moment vector on the left-hand-side.

A similar argument can be used to show that, for any fixed $l$, the pixel intensities can be reconstructed from the moment vector $\left\{\mu_{j, l}\right\}_{j=0}^{N-1}$, which can be obtained from the Pascal triangle $T^{N+l-1}(I)$ of order $N+l-1$.

Remark 1. In practice, when reconstructing the pixel intensities of an image $I$, floating point errors in the matrix inversion can result in inaccuracies in the reconstructed image. In fact, the recovered pixel intensities may be complex valued. While the imaginary part of the result tends to be quite small, it is advantageous to first reformulate the problem to guarantee a real solution. One way to force the solution to be real is to separate equation (2) into two sets of equations with real coefficients. More specifically, we can separate the equation system into its real part and its imaginary part, and combine these two real equation systems into one. After this, a real solution for the new equation system can be found, for example, by singular value decomposition (SVD).

Lemma 2. Given the moments matrix $\tau_{N}(I)$ of a discrete image $I$ and an upper bound on the number $N$ of pixels, one can reconstruct the pixel location $z_{k}$ and the intensity $\rho\left(z_{k}\right)$ for all $z_{k}$ such that $\rho\left(z_{k}\right) \neq 0 .{ }^{3}$

Proof. If the number of pixels in the image $I$ is strictly less than $N$, we can extend $I$ to an image with $N$ pixels by adding zero intensity pixels. Without loss of generality, we assume that $\rho\left(z_{k}\right) \neq 0$ for $k=1, \ldots, s$ and $\rho\left(z_{k}\right)=0$ for $k=s+1, \ldots, N$. Consider the polynomial

$$
P(t)=\prod_{k=1}^{s}\left(t-z_{k}\right)=t^{s}+\sum_{j=1}^{s} c_{j} t^{s-j},
$$

where the coefficients $c_{j}$ are polynomials in the $z_{k}$ 's.

\footnotetext{
${ }^{3}$ This result generalizes Proposition 1 in [6], which states that the vertices of a polygon are uniquely determined by a finite number of moments.
} 
Observe that $P\left(z_{k}\right)=0, \forall k=1,2, \ldots, s$. Therefore, we also have $\rho\left(z_{k}\right) \bar{z}_{k}^{l} P\left(z_{k}\right)=0$, for any $l=0, \ldots, s-1$. Summing all these equations over $k$ 's, we get

$$
\begin{aligned}
\sum_{k=1}^{s} \rho\left(z_{k}\right) \bar{z}_{k}^{l} P\left(z_{k}\right)=0 & \Longrightarrow \sum_{k=1}^{s} \rho\left(z_{k}\right) \bar{z}_{k}^{l}\left(z_{k}^{s}+\sum_{j=1}^{s} c_{j} z_{k}^{s-j}\right)=0 \\
& \Longrightarrow \sum_{k=1}^{s} \rho\left(z_{k}\right) \bar{z}_{k}^{l} z_{k}^{s}+\sum_{j=1}^{s} c_{j} \sum_{k=1}^{s} \rho\left(z_{k}\right) \bar{z}_{k}^{l} z_{k}^{s-j}=0 \\
& \Longrightarrow \mu_{s, l}+\sum_{j=1}^{s} c_{j} \mu_{s-j, l}=0 \\
& \Longrightarrow \sum_{j=1}^{s} c_{j} \mu_{s-j, l}=-\mu_{s, l}, \quad l=0,1, \ldots, s-1 .
\end{aligned}
$$

We write these last equations in matrix form:

$$
\underbrace{\left(\begin{array}{cccccc}
\mu_{0,0} & \mu_{1,0} & \mu_{2,0} & \mu_{3,0} & \cdots & \mu_{s-1,0} \\
\mu_{0,1} & \mu_{1,1} & \mu_{2,1} & \cdots & \cdots & \mu_{s-1,1} \\
\mu_{0,2} & \mu_{1,2} & \cdots & \cdots & \cdots & \mu_{s-1,2} \\
\mu_{0,3} & \cdots & \cdots & \cdots & \cdots & \mu_{s-1,3} \\
\vdots & & & & & \vdots \\
\mu_{0, s-1} & \mu_{1, s-1} & \cdots & \cdots & \cdots & \mu_{s-1, s-1}
\end{array}\right)}_{\tau_{s}(I)}\left(\begin{array}{c}
c_{s} \\
c_{s-1} \\
c_{s-2} \\
\vdots \\
c_{1}
\end{array}\right)=-\left(\begin{array}{c}
\mu_{s, 0} \\
\mu_{s, 1} \\
\mu_{s, 2} \\
\vdots \\
\mu_{s, s-1}
\end{array}\right) .
$$

From equation (1) we know that

$$
\tau_{s}(I)=\left(\begin{array}{cccc}
1 & 1 & \cdots & 1 \\
\bar{z}_{1} & \bar{z}_{2} & \cdots & \bar{z}_{s} \\
\vdots & \cdots & \vdots & \\
\bar{z}_{1}^{s-1} & \bar{z}_{2}^{s-1} & \cdots & \bar{z}_{s}^{s-1}
\end{array}\right)\left(\begin{array}{cccc}
\rho\left(z_{1}\right) & 0 & \cdots & 0 \\
0 & \rho\left(z_{2}\right) & \cdots & 0 \\
\vdots & \vdots & \ddots & \vdots \\
0 & \cdots & 0 & \rho\left(z_{s}\right)
\end{array}\right)\left(\begin{array}{ccccc}
1 & z_{1} & z_{1}^{2} & \cdots & z_{1}^{s-1} \\
1 & z_{2} & z_{2}^{2} & \cdots & z_{2}^{s-1} \\
\vdots & \vdots & \cdots & \vdots & \\
1 & z_{s} & z_{s}^{2} & \cdots & z_{s}^{s-1}
\end{array}\right) .
$$

Thus $\tau_{s}(I)$ is invertible, since the locations $z_{k}$ are pairwise distinct and the pixel intensities $\rho\left(z_{k}\right)$ are nonzero. Hence we can solve the above equation system for $\left(c_{s}, c_{s-1}, \ldots, c_{1}\right)$ by inverting $\tau_{s}(I)$ and multiplying by the vector on the right-hand-side of equation (4).

Since the $c_{k}$ 's determine the polynomial $P(t)$, we can solve for the roots of $P(t)=0$, which are actually $\left\{z_{k}\right\}_{k=1}^{s}$. By Lemma 1 , we can subsequently obtain the pixel intensities $\left\{\rho\left(z_{k}\right)\right\}_{k=1}^{s}$.

Remark 2. To determine the number of nonzero pixels, we can look at the rank of $\tau_{N}(I)$. Since $\tau_{N}(I)=Z^{\dagger} W Z$ by equation (1) and $\operatorname{rank}\left(Z^{\dagger}\right)=\operatorname{rank}(Z)=N, \operatorname{rank}(W)=s$, we can conclude that $\operatorname{rank}\left(\tau_{N}(I)\right)=s$.

Since the Pascal triangle $T^{2 N-2}(I)$ of the image $I$ contains all the information needed to recover $\tau_{N}(I)$, we have the following corollary:

Corollary 1 (image reconstruction property). Given the Pascal triangle $T^{2 N-2}(I)$ of a discrete image $I$, one can reconstruct both the grid point locations $\left\{z_{k}\right\}_{k=1}^{N}$ and the corresponding intensities $\left\{\rho\left(z_{k}\right)\right\}_{k=1}^{N}$ for all those $z_{k}$ such that $\rho\left(z_{k}\right) \neq 0$. 


\section{Relationship with the Radon transform}

The Radon transform $f_{\theta}(r)$ is the projection of the image $I=\left\{\left(z_{k}, \rho\left(z_{k}\right)\right)\right\}_{k=1}^{N}$ onto the straight line through the origin with direction vector $(\cos (\theta) \sin (\theta))^{T}$, i.e.

$$
f_{\theta}(r)=\sum_{k \in S} \rho\left(z_{k}\right)
$$

where $S=\left\{k \mid x_{k} \cos (\theta)+y_{k} \sin (\theta)=r, k=1,2, \ldots, N\right\}$. Since $f_{\theta}(r)$ is a periodic function of $\theta$ with period $2 \pi$, any of its $n$-th order moment $m_{n}(\theta)$ is also periodic with period $2 \pi$. It turns out that, for any $n=0,1,2, \ldots$, the coefficients of the Fourier series of $m_{n}(\theta)$ are given by the entries of row $(n+1)$ of $T^{r}(I)$ with $r \geq n$.

Lemma 3. The $n$-th order moment $m_{n}(\theta)$ of the Radon transform $f_{\theta}(r)$ is given by the following linear combination of the $(n+1)$-th row entries of the Pascal triangle $T^{r}(I)$ with $r \geq n$ :

$$
m_{n}(\theta)=\frac{1}{2^{n}} \sum_{l=0}^{n}\left(\begin{array}{l}
n \\
l
\end{array}\right) \mu_{l, n-l} e^{i(n-2 l) \theta} .
$$

Proof. For $n=0$, we have

$$
m_{0}(\theta)=\sum_{k=1}^{N} \rho\left(z_{k}\right)=\sum_{k=1}^{N} \rho\left(z_{k}\right) z_{k}^{0} \bar{z}_{k}^{0}=\mu_{0,0} .
$$

For $n>0$, we have

$$
m_{n}(\theta)=\sum_{r} r^{n} f_{\theta}(r)=\sum_{k=1}^{N}\left(r_{k}(\theta)\right)^{n} \rho\left(z_{k}\right),
$$

where $r_{k}(\theta)$ is the projection of the vector $\left(x_{k}, y_{k}\right)^{T}$ onto the axis with angle $\theta \in(-\pi, \pi]$ with respect to $x$-axis. More precisely,

$$
r_{k}(\theta)=x_{k} \cos \theta+y_{k} \sin \theta=\frac{1}{2}\left(z_{k} e^{-i \theta}+\bar{z}_{k} e^{i \theta}\right), \quad \forall k=1,2, \ldots, N,
$$

and therefore

$$
\begin{aligned}
m_{n}(\theta) & =\sum_{k=1}^{N}\left(r_{k}(\theta)\right)^{n} \rho\left(z_{k}\right)=\sum_{k=1}^{N}\left(\frac{1}{2}\left(z_{k} e^{-i \theta}+\bar{z}_{k} e^{i \theta}\right)\right)^{n} \rho\left(z_{k}\right) \\
& =\frac{1}{2^{n}} \sum_{k=1}^{N}\left(z_{k} e^{-i \theta}+\bar{z}_{k} e^{i \theta}\right)^{n} \rho\left(z_{k}\right)=\frac{1}{2^{n}} \sum_{k=1}^{N}\left(\sum_{l=0}^{n}\left(\begin{array}{c}
n \\
l
\end{array}\right) z_{k}^{l} e^{-i l \theta} \bar{z}_{k}^{n-l} e^{i(n-l) \theta}\right) \rho\left(z_{k}\right) \\
& =\frac{1}{2^{n}} \sum_{l=0}^{n}\left(\begin{array}{c}
n \\
l
\end{array}\right)\left(\sum_{k=1}^{N} z_{k}^{l} \bar{z}_{k}^{n-l} \rho\left(z_{k}\right)\right) e^{-i l \theta} e^{i(n-l) \theta}=\frac{1}{2^{n}} \sum_{l=0}^{n}\left(\begin{array}{c}
n \\
l
\end{array}\right) \mu_{l, n-l} e^{i(n-2 l) \theta} .
\end{aligned}
$$

Figs. 1 and 2 summarize the relationship between the Pascal triangle and the Radon transform of an image when the pixel locations are known and unknown, respectively. Observe that a smaller number of rows of the Pascal triangle are needed in order to reconstruct the image if the pixel locations were known. 


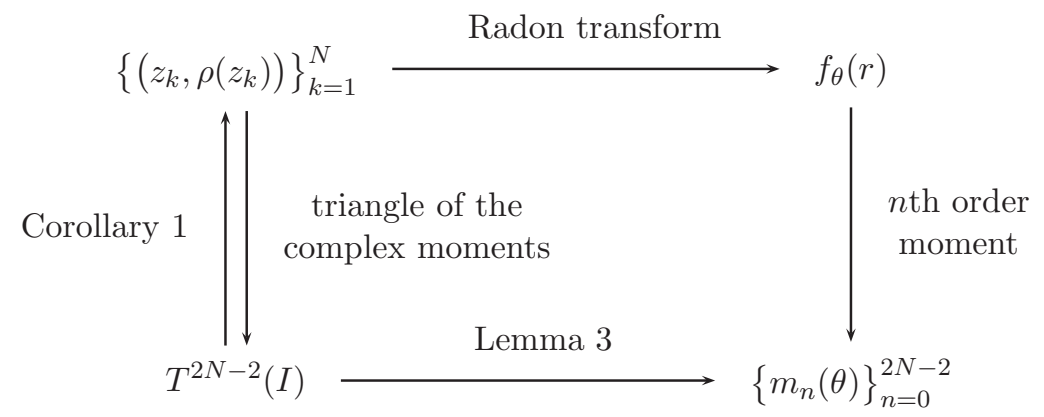

Figure 1. Relationship between the Pascal triangle and the Radon transform of an image under the assumption that the pixel locations are unknown a priori.

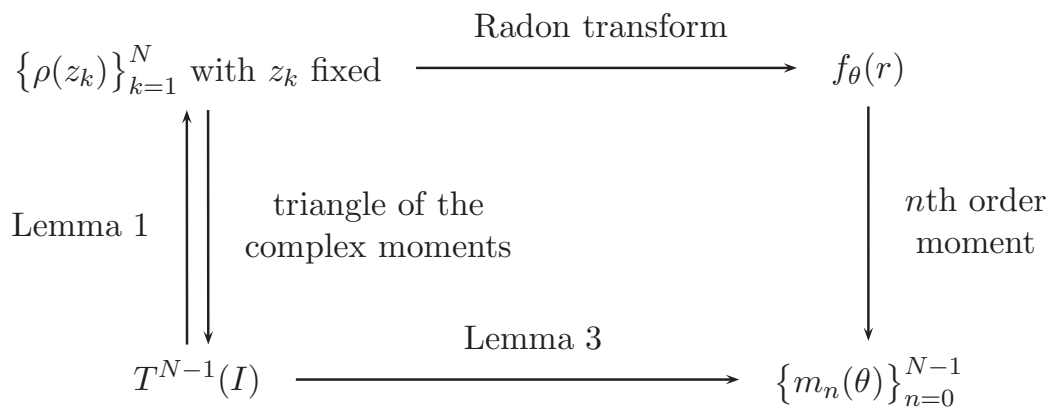

Figure 2. Relationship between the Pascal triangle and the Radon transform of an image under the assumption that the pixel locations are fixed and known a priori.

\section{Image reconstruction from samples}

Lemma 4. The last row of $T^{n}(I)$ can be reconstructed from $n+1$ generic moment samples $m_{n}\left(\theta_{1}\right), m_{n}\left(\theta_{2}\right), \ldots, m_{n}\left(\theta_{n+1}\right)$.

Proof. We first write equation (5) in matrix form:

$$
\left(\begin{array}{c}
m_{n}\left(\theta_{1}\right) \\
m_{n}\left(\theta_{2}\right) \\
\vdots \\
m_{n}\left(\theta_{n+1}\right)
\end{array}\right)=\frac{1}{2^{n}}\left(\begin{array}{ccccc}
e^{i n \theta_{1}} & \cdots & e^{i(n-2 l) \theta_{1}} & \cdots & e^{-i n \theta_{1}} \\
e^{i n \theta_{2}} & \cdots & e^{i(n-2 l) \theta_{2}} & \cdots & e^{-i n \theta_{2}} \\
\vdots & \cdots & \vdots & \cdots & \vdots \\
e^{i n \theta_{n+1}} & \cdots & e^{i(n-2 l) \theta_{n+1}} & \cdots & e^{-i n \theta_{n+1}}
\end{array}\right)\left(\begin{array}{c}
\mu_{0, n} \\
\vdots \\
\left(\begin{array}{c}
n \\
l
\end{array}\right) \mu_{l, n-l} \\
\vdots \\
\mu_{n, 0}
\end{array}\right)
$$

There is a unique solution for $\left(\mu_{0, n}, \ldots,\left(\begin{array}{c}n \\ l\end{array}\right) \mu_{l, n-l}, \ldots, \mu_{n, 0}\right)$ if and only if

$$
\begin{aligned}
& \operatorname{det}\left[\left(\begin{array}{ccccc}
e^{i n \theta_{1}} & \cdots & e^{i(n-2 l) \theta_{1}} & \cdots & e^{-i n \theta_{1}} \\
e^{i n \theta_{2}} & \cdots & e^{i(n-2 l) \theta_{2}} & \cdots & e^{-i n \theta_{2}} \\
\vdots & \cdots & \vdots & \cdots & \vdots \\
e^{i n \theta_{n+1}} & \cdots & e^{i(n-2 l) \theta_{n+1}} & \cdots & e^{-i n \theta_{n+1}}
\end{array}\right)\right] \neq 0 \\
& \Longleftrightarrow \operatorname{det}\left[\left(\begin{array}{ccccc}
e^{-i n \theta_{1}} & 0 & 0 & \ldots & 0 \\
0 & e^{-i n \theta_{2}} & 0 & \cdots & 0 \\
\vdots & \vdots & \ddots & & \vdots \\
0 & \cdots & 0 & \ldots & e^{-i n \theta_{n+1}}
\end{array}\right)\left(\begin{array}{ccccc}
e^{i 2 n \theta_{1}} & \cdots & e^{i 4 \theta_{1}} & e^{i 2 \theta_{1}} & 1 \\
e^{i 2 n \theta_{2}} & \cdots & e^{i 4 \theta_{2}} & e^{i 2 \theta_{2}} & 1 \\
\vdots & \cdots & \vdots & \vdots & \vdots \\
e^{i 2 n \theta_{n+1}} & \cdots & e^{i 4 \theta_{n+1}} & e^{i 2 \theta_{n+1}} & 1
\end{array}\right)\right] \neq 0
\end{aligned}
$$




$$
\Longleftrightarrow \operatorname{det}\left[\left(\begin{array}{ccccc}
e^{i 2 n \theta_{1}} & \cdots & e^{i 4 \theta_{1}} & e^{i 2 \theta_{1}} & 1 \\
e^{i 2 n \theta_{2}} & \cdots & e^{i 4 \theta_{2}} & e^{i 2 \theta_{2}} & 1 \\
\vdots & \cdots & \vdots & \vdots & \vdots \\
e^{i 2 n \theta_{n+1}} & \cdots & e^{i 4 \theta_{n+1}} & e^{i 2 \theta_{n+1}} & 1
\end{array}\right)\right] \neq 0
$$

since $e^{-i n \theta_{j}} \neq 0, \forall j=1, \ldots, n+1$.

Observe that the above determinant is a Vandermonde determinant. It is nonzero if $e^{i 2 \theta_{j}} \neq$ $e^{i 2 \theta_{k}}$ for all distinct $j, k=1, \ldots, n+1$. Therefore, if the $\theta_{j}$ 's are such that $e^{i 2 \theta_{j}} \neq e^{i 2 \theta_{k}}$ (thus the need to pick a generic sample set), we will get a unique solution for $\left(\mu_{0, n}, \ldots,\left(\begin{array}{c}n \\ l\end{array}\right) \mu_{l, n-l}, \ldots, \mu_{n, 0}\right)$. Hence we can reconstruct the last row of $T^{n}(I)$.

Corollary 2. Given the grid point locations $\left\{z_{k}\right\}_{k=1}^{N}$, we can reconstruct the discrete image $I=$ $\left\{\left(z_{k}, \rho\left(z_{k}\right)\right)\right\}_{k=1}^{N}$ from the Radon transform $f_{\theta_{1}}(r), f_{\theta_{2}}(r), \ldots, f_{\theta_{N}}(r)$ at $N$ fixed generic angles $\theta_{1}, \ldots, \theta_{N} \cdot{ }^{4}$

Proof. From the given radon transform of the image at different angles, we can calculate the moments $\left\{m_{n}\left(\theta_{k}\right) \mid n=0, \ldots, N-1, k=1, \ldots, n+1\right\}$. The conclusion followed by combining Lemmas 1 and 4.

The diagram of Fig. 3 thus commutes. Note that, one could use a similar argument along with Corollary 1 to show that $(2 N-1)$ generic observations of $\left\{m_{n}\left(\theta_{j}\right), j=1, \ldots, n+1\right\}_{n=0}^{2 N-2}$ would be needed to fully reconstruct the image $\left\{\left(z_{k}, \rho\left(z_{k}\right)\right)\right\}_{k=1}^{N}$ with pixel positions $z_{k}$ unknown.

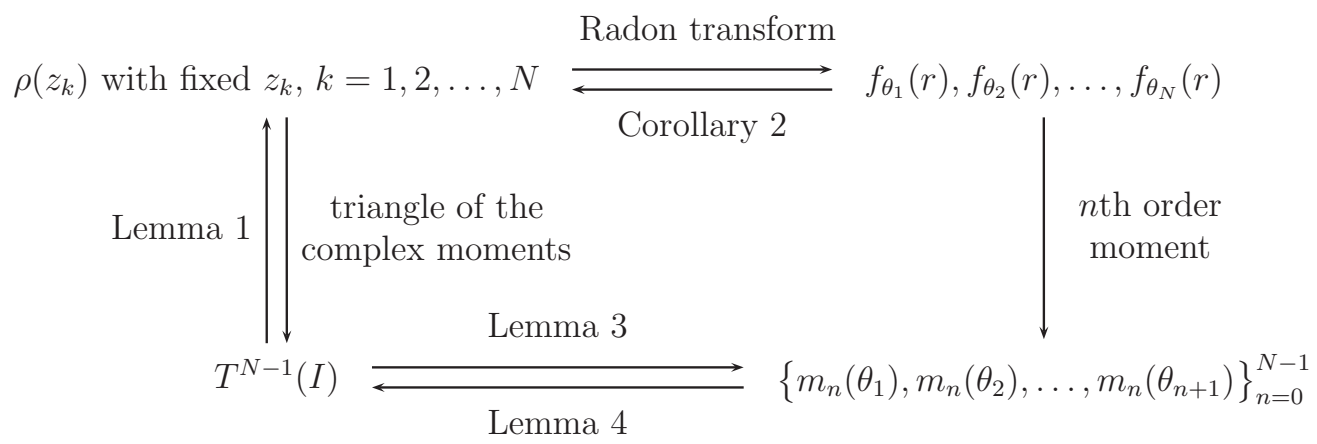

Figure 3. Relationship between the Pascal triangle $T^{N-1}(I)$ and the Radon transform.

\section{Prolongation of group actions on the moments and invariantization}

Let $(G, \cdot)$ be a group acting on the complex plane:

$$
\begin{aligned}
\because G \times \mathbb{C} & \longrightarrow \mathbb{C}, \\
(g, z) & \longmapsto g \cdot z, \quad \forall g \in G, z \in \mathbb{C} .
\end{aligned}
$$

This induces a group transformation $(G, \circ)$ of the discrete image $I=\left\{\left(z_{k}, \rho\left(z_{k}\right)\right)\right\}_{k=1}^{N}$, namely

$$
g \circ\left\{\left(z_{k}, \rho\left(z_{k}\right)\right)\right\}_{k=1}^{N}=\left\{\left(g \cdot z_{k}, \rho\left(z_{k}\right)\right)\right\}_{k=1}^{N}, \quad \forall g \in G .
$$

\footnotetext{
${ }^{4}$ This result generalizes Theorem 5.1 in [4], which states that a quadrature domain can be uniquely reconstructed by the line integral projections at finite angles.
} 
Then the induced transformation $(G, *)$ on moments $\left\{\mu_{j, l}\right\}_{j, l \in \mathbb{Z}_{\geq} 0}$ is

$$
g *\left\{\mu_{j, l}\right\}_{j, l \in \mathbb{Z}_{\geq} 0}=g *\left\{\sum_{k=1}^{N} z_{k}^{j} \bar{z}_{k}^{l} \rho\left(z_{k}\right)\right\}_{j, l \in \mathbb{Z}_{\geq} 0}=\left\{\sum_{k=1}^{N}\left(g \cdot z_{k}\right)^{j}\left(\overline{g \cdot z_{k}}\right)^{l} \rho\left(z_{k}\right)\right\}_{j, l \in \mathbb{Z}_{\geq} 0} .
$$

In other words, the transformed moments are the moments of the transformed image.

Example 1. Consider the action of $G=\mathbb{C}$ on $\mathbb{C}$ by translation

$$
\left(z_{0}, z\right) \mapsto z+z_{0}, \quad \forall z_{0} \in G, \quad \forall z \in \mathbb{C} .
$$

Then the induced transformation on the image $I=\left\{\left(z_{k}, \rho\left(z_{k}\right)\right)\right\}_{k=1}^{N}$ is

$$
z_{0} \circ\left\{\left(z_{k}, \rho\left(z_{k}\right)\right)\right\}_{k=1}^{N}=\left\{\left(z_{k}+z_{0}, \rho\left(z_{k}\right)\right)\right\}_{k=1}^{N}, \quad \forall z_{0} \in G .
$$

In other words, the image is translated horizontally with distance $x_{0}=\operatorname{Re}\left(z_{0}\right)$ and vertically with $y_{0}=\operatorname{Im}\left(z_{0}\right)$. The transformed complex moments are

$$
\begin{aligned}
\tilde{\mu}_{j, l} & =\sum_{k=1}^{N} \tilde{z}_{k}^{j} \bar{z}_{k}^{l} \rho\left(\tilde{z}_{k}\right)=\sum_{k=1}^{N}\left(z_{k}+z_{0}\right)^{j}\left(\bar{z}_{k}+\bar{z}_{0}\right)^{l} \rho\left(z_{k}\right) \\
& =\sum_{k=1}^{N}\left(\sum_{s=0}^{j}\left(\begin{array}{l}
j \\
s
\end{array}\right) z_{k}^{s} z_{0}^{j-s}\right)\left(\sum_{t=0}^{l}\left(\begin{array}{l}
l \\
t
\end{array}\right) \bar{z}_{k}^{t} \bar{z}_{0}^{l-t}\right) \rho\left(z_{k}\right) \\
& =\sum_{s=0}^{j} \sum_{t=0}^{l}\left(\sum_{k=1}^{N} z_{k}^{s} \bar{z}_{k}^{t} \rho\left(z_{k}\right)\right)\left(\begin{array}{l}
j \\
s
\end{array}\right)\left(\begin{array}{l}
l \\
t
\end{array}\right) z_{0}^{j-s} \bar{z}_{0}^{l-t} \\
& =\sum_{s=0}^{j} \sum_{t=0}^{l} \mu_{s, t}\left(\begin{array}{l}
j \\
s
\end{array}\right)\left(\begin{array}{l}
l \\
t
\end{array}\right) z_{0}^{j-s} \bar{z}_{0}^{l-t}, \quad \forall j, l \in \mathbb{Z}_{\geq 0} .
\end{aligned}
$$

Written in matrix form, the transformation of the moment matrix $\tau_{N}(I)$ is $\tau_{N}(\tilde{I})=A^{\dagger} \tau_{N}(I) A$, where $A=\left(a_{j, l}\right)_{N \times N}$ is an upper-triangular matrix with $a_{j, l}=\left(\begin{array}{c}l-1 \\ j-1\end{array}\right) z_{0}^{l-j}$, and $A^{\dagger}$ is the conjugate transpose of $A$.

Having obtained an explicit formula for the action of $G$ on the moments, we follow Fels and Olver's moving frame method [1,2, 7] to obtain a set of invariant functions of the moments. More specifically, we consider the cross-section defined by $\tilde{\mu}_{1,0}=0$. The group transformation that maps $\tau_{N}(I)$ to the cross-section is the moving frame $z_{0}=-\frac{\mu_{1,0}}{\mu_{0,0}}$. By applying the moving frame to the moment matrix, we obtain the matrix $\tilde{\tau}_{N}(I)=A_{0}^{\dagger} \tau_{N}(I) A_{0}$, where $A_{0}=$ $\left(\left(\begin{array}{l}l-1 \\ j-1\end{array}\right)\left(-\frac{\mu_{1,0}}{\mu_{0,0}}\right)^{l-j}\right)_{N \times N}$. By equivariance of the moving frame, all the entries of $\tilde{\tau}_{N}(I)$ are invariant under translation. One can check that theses entries $\tilde{\mu}_{j, l}$ are actually the centralized moments

$$
\tilde{\mu}_{j, l}=\sum_{k=1}^{N}\left(z_{k}-\frac{\mu_{1,0}}{\mu_{0,0}}\right)^{j}\left(\bar{z}_{k}-\frac{\bar{\mu}_{1,0}}{\mu_{0,0}}\right)^{l} \rho\left(z_{k}\right), \quad j, l \in \mathbb{Z}_{\geq 0} .
$$

By normalizing (i.e. applying the moving frame transformation to) the coordinates of $T^{r}(I)$, we obtain the translation invariant Pascal triangle $T_{\text {trans }}^{r}(I)$ for a discrete image $I$ :

$$
\begin{aligned}
& \tilde{\mu}_{0,0} \\
& \begin{array}{cccc}
\tilde{\mu}_{0,2} & \tilde{\mu}_{0,1} & \tilde{\mu}_{1,0} & \\
& 2 \tilde{\mu}_{1,1} & \tilde{\mu}_{2,0}
\end{array}
\end{aligned}
$$

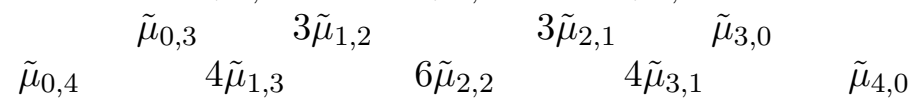

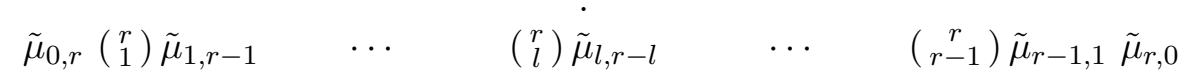


Observe that the corresponding $n$-th order central moment $\tilde{m}_{n}(\theta)$ of the image,

$$
\tilde{m}_{n}(\theta)=\sum_{k=1}^{N}\left(r_{k}(\theta)-r_{0}(\theta)\right)^{n} \rho\left(x_{k}, y_{k}\right) \text {, }
$$

where $r_{0}(\theta)=x_{0} \cos \theta+y_{0} \sin \theta$ is the projection of the centroid, is invariant under translations.

Lemma 5 (orbit separation property of $T_{\text {trans }}^{2 N-2}(I)$ ). Let $I_{1}, I_{2}$ be two discrete gray scale images with the same number $N$ of pixels. There exists a translation $g \in \mathbb{C}$ such that $g \circ I_{1}=I_{2}$, where $\circ$ is defined as in $(6) \Longleftrightarrow T_{\text {trans }}^{r}\left(I_{1}\right)=T_{\text {trans }}^{r}\left(I_{2}\right)$ for $r \geq 2 N-2$.

Proof. $\Rightarrow$ If $\exists g \in G$ such that $g \circ I_{1}=I_{2}$, we have $z_{k}^{(2)}=z_{k}^{(1)}+z_{0}$ and $\rho_{2}\left(z_{k}^{(2)}\right)=\rho_{1}\left(z_{k}^{(1)}\right)$, for some $z_{0} \in \mathbb{C}, k=1, \ldots, N$. From equation (7) we know that

$$
\mu_{0,0}^{(2)}=\mu_{0,0}^{(1)}, \quad \mu_{1,0}^{(2)}=\mu_{0,0}^{(1)} z_{0}+\mu_{1,0}^{(1)}, \quad \text { hence } \quad \frac{\mu_{1,0}^{(2)}}{\mu_{0,0}^{(2)}}=\frac{\mu_{1,0}^{(1)}}{\mu_{0,0}^{(1)}}+z_{0} .
$$

Then applying equation (8), we can get for any $j, l \in \mathbb{Z}_{\geq 0}$

$$
\begin{aligned}
\tilde{\mu}_{j, l}^{(1)} & =\sum_{k=1}^{N}\left(z_{k}^{(1)}-\frac{\mu_{1,0}^{(1)}}{\mu_{0,0}^{(1)}}\right)^{j}\left(\bar{z}_{k}^{(1)}-\frac{\bar{\mu}_{1,0}^{(1)}}{\mu_{0,0}^{(1)}}\right)^{l} \rho_{1}\left(z_{k}^{(1)}\right) \\
& =\sum_{k=1}^{N}\left(z_{k}^{(2)}-\frac{\mu_{1,0}^{(1)}}{\mu_{0,0}^{(1)}}-z_{0}\right)^{j}\left(\bar{z}_{k}^{(2)}-\frac{\bar{\mu}_{1,0}^{(1)}}{\mu_{0,0}^{(1)}}-\bar{z}_{0}\right)^{l} \rho_{2}\left(z_{k}^{(2)}\right)=\tilde{\mu}_{j, l}^{(2)} .
\end{aligned}
$$

Therefore $T_{\text {trans }}^{r}\left(I_{1}\right)=T_{\text {trans }}^{r}\left(I_{2}\right)$ for any $r \in \mathbb{Z}_{\geq 0}$. i.e.

$\Leftarrow$ If $T_{\text {trans }}^{r}\left(I_{1}\right)=T_{\text {trans }}^{r}\left(I_{2}\right)$ for $r \geq 2 N-2$, from Corollary 1 , we conclude that $I_{1}^{\text {trans }}=I_{2}^{\text {trans }}$,

$$
\left\{\left(z_{k, \text { trans }}^{(1)}, \rho_{1}\left(z_{k, \text { trans }}^{(1)}\right)\right)\right\}_{k=1}^{N}=\left\{\left(z_{k, \text { trans }}^{(2)}, \rho_{2}\left(z_{k, \text { trans }}^{(2)}\right)\right)\right\}_{k=1}^{N}
$$

Hence $\exists z_{0}, z_{0}^{\prime} \in \mathbb{C}$ s.t. $z_{k \text {,trans }}^{(1)}=z_{k}^{(1)}+z_{0}, z_{k \text {,trans }}^{(2)}=z_{k}^{(2)}+z_{0}^{\prime}$ with $\rho_{1}\left(z_{k, \text { trans }}^{(1)}\right)=\rho_{1}\left(z_{k}^{(1)}\right)$ and $\rho_{2}\left(z_{k, \text { trans }}^{(2)}\right)=\rho_{2}\left(z_{k}^{(2)}\right)$ for any $k=1,2, \ldots, N$. Without loss of generality, we assume that $z_{k, \text { trans }}^{(1)}=z_{k \text {,trans }}^{(2)}$ and $\rho_{1}\left(z_{k, \text { trans }}^{(1)}\right)=\rho_{2}\left(z_{k, \text { trans }}^{(2)}\right)$. Therefore $\exists z_{0}-z_{0}^{\prime} \in \mathbb{C}=G$ satisfying

$$
z_{k}^{(1)}+z_{0}-z_{0}^{\prime}=z_{k}^{(2)}, \quad \rho_{1}\left(z_{k}^{(1)}+\left(z_{0}-z_{0}^{\prime}\right)\right)=\rho_{1}\left(z_{k}^{(1)}\right)=\rho_{2}\left(z_{k}^{(2)}\right),
$$

i.e. $\exists g=z_{0}^{\prime}-z_{0} \in G=\mathbb{C}$ such that $g \circ I_{1}=I_{2}$.

Remark 3. Without loss of generality, we can assume that the two images have the same number of pixels by simply adding zero valued pixels to the smaller image.

Example 2. Consider the action of $G=\mathbb{R}_{+}$on $\mathbb{C}$ by scaling

$$
(\lambda, z) \mapsto \lambda z, \quad \forall \lambda \in G, \quad \forall z \in \mathbb{C} .
$$

Then the induced transformation on the image $I=\left\{\left(z_{k}, \rho\left(z_{k}\right)\right)\right\}_{k=1}^{N}$ is

$$
\lambda \circ\left\{\left(z_{k}, \rho\left(z_{k}\right)\right)\right\}_{k=1}^{N}=\left\{\left(\lambda z_{k}, \rho\left(z_{k}\right)\right)\right\}_{k=1}^{N}, \quad \forall \lambda \in G .
$$


In other words, the image is scaled by a factor $\lambda$ both horizontally and vertically. Then the transformed complex moments are

$$
\begin{aligned}
\hat{\mu}_{j, l} & =\sum_{k=1}^{N} \hat{z}_{k}^{j} \overline{\hat{z}}_{k}^{l} \rho\left(\hat{z}_{k}\right)=\sum_{k=1}^{N}\left(\lambda z_{k}\right)^{j}\left(\lambda \bar{z}_{k}\right)^{l} \rho\left(z_{k}\right) \\
& =\sum_{k=1}^{N} \lambda^{j+l} z_{k}^{j} \bar{z}_{k}^{l} \rho\left(z_{k}\right)=\lambda^{j+l} \sum_{k=1}^{N} z_{k}^{j} \bar{z}_{k}^{l} \rho\left(z_{k}\right)=\mu_{j, l} \lambda^{j+l}, \quad \forall j, l \in \mathbb{Z}_{\geq 0} .
\end{aligned}
$$

Written in matrix form, the moment matrix for the new image $\hat{I}$ after scaling is

$$
\tau_{N}(\hat{I})=\left(\begin{array}{ccccc}
1 & 0 & 0 & \cdots & 0 \\
0 & \lambda & 0 & \cdots & 0 \\
0 & 0 & \lambda^{2} & \cdots & 0 \\
\vdots & \vdots & \vdots & \ddots & \vdots \\
0 & \cdots & \cdots & 0 & \lambda^{N-1}
\end{array}\right) \tau_{N}(I)\left(\begin{array}{ccccc}
1 & 0 & 0 & \cdots & 0 \\
0 & \lambda & 0 & \cdots & 0 \\
0 & 0 & \lambda^{2} & \cdots & 0 \\
\vdots & \vdots & \vdots & \ddots & \vdots \\
0 & \cdots & \cdots & 0 & \lambda^{N-1}
\end{array}\right) .
$$

Again, we use the moving frame method of Fels and Olver to obtain a set of invariant functions of the moments. Notice that $\hat{\mu}_{1,1}=\sum_{k=1}^{N} \hat{z}_{k} \overline{\hat{z}}_{k} \rho\left(\hat{z}_{k}\right)=\sum_{k=1}^{N}\left(\hat{x}_{k}^{2}+\hat{y}_{k}^{2}\right) \rho\left(\hat{z}_{k}\right) \neq 0$ unless all $\rho\left(z_{k}\right)$ are zero. We consider the cross-section defined by $\hat{\mu}_{1,1}=1$. The group transformation that maps $\tau_{N}(I)$ to the cross-section is the moving frame $\lambda=\left(\mu_{1,1}\right)^{-\frac{1}{2}}$. By applying the moving frame to the moment matrix, we obtain the matrix

$$
\hat{\tau}_{N}(I)=\left(\begin{array}{cccc}
1 & 0 & \cdots & 0 \\
0 & \left(\mu_{1,1}\right)^{-\frac{1}{2}} & \cdots & 0 \\
\vdots & \vdots & \ddots & \vdots \\
0 & \cdots & 0 & \left(\mu_{1,1}\right)^{-\frac{N-1}{2}}
\end{array}\right) \tau_{N}(I)\left(\begin{array}{cccc}
1 & 0 & \cdots & 0 \\
0 & \left(\mu_{1,1}\right)^{-\frac{1}{2}} & \cdots & 0 \\
\vdots & \vdots & \ddots & \vdots \\
0 & \cdots & 0 & \left(\mu_{1,1}\right)^{-\frac{N-1}{2}}
\end{array}\right)
$$

By equivariance of the moving frame, all the entries of $\hat{\tau}_{N}(I)$ are invariant under scaling.

By normalizing the coordinates of $T^{r}(I)$, we obtain the scaling invariant Pascal triangle $T_{\text {scale }}^{r}(I)$ for a discrete image $I$ :

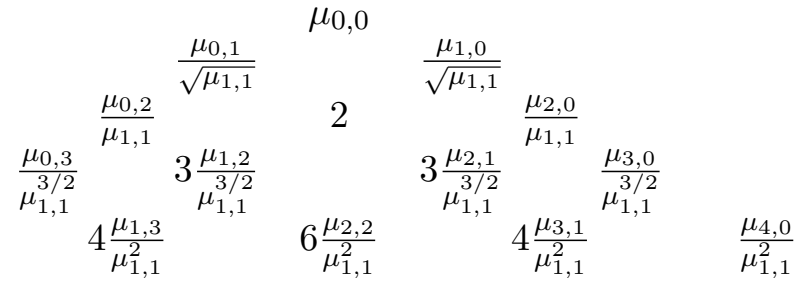

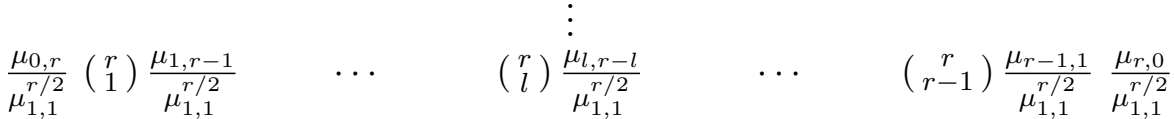

Observe that the corresponding $n$-th order normalized moment $\hat{m}_{n}(\theta)=m_{n}(\theta) / \mu_{1,1}^{\frac{n}{2}}$ is invariant under scaling.

Lemma 6 (orbit separation property of $T_{\text {scale }}^{2 N-2}(I)$ ). Let $I_{1}, I_{2}$ be two discrete gray scale images with the same number $N$ of pixels. There exists a scaling $g \in \mathbb{R}_{+}$such that $g \circ I_{1}=I_{2}$, where $\circ$ is defined as in $(9) \Longleftrightarrow T_{\text {scale }}^{r}\left(I_{1}\right)=T_{\text {scale }}^{r}\left(I_{2}\right)$ for $r \geq 2 N-2$. 
Proof. $\Rightarrow$ If $\exists g \in G$ such that $g \circ I_{1}=I_{2}$, we have $z_{k}^{(2)}=\lambda z_{k}^{(1)}$ and $\rho_{1}\left(z_{k}^{(1)}\right)=\rho_{2}\left(z_{k}^{(2)}\right)$ for some $\lambda \in \mathbb{R}_{+}, k=1, \ldots, N$. Since for $I_{1}$ and $I_{2}$, the corresponding scaling invariant moments are

$$
\begin{aligned}
\hat{\mu}_{j, l}^{(1)}= & \frac{\mu_{j, l}^{(1)}}{\left(\mu_{1,1}^{(1)}\right)^{\frac{j+l}{2}}}=\frac{\sum_{k=1}^{N}\left(z_{k}^{(1)}\right)^{j}\left(\bar{z}_{k}^{(1)}\right)^{l} \rho_{1}\left(z_{k}^{(1)}\right)}{\left(\sum_{k=1}^{N} z_{k}^{(1)} \bar{z}_{k}^{(1)} \rho_{1}\left(z_{k}^{(1)}\right)\right)^{\frac{j+l}{2}}}, \\
\hat{\mu}_{j, l}^{(2)}= & \frac{\mu_{j, l}^{(2)}}{\left(\mu_{1,1}^{(2)}\right)^{\frac{j+l}{2}}}=\frac{\sum_{k=1}^{N}\left(z_{k}^{(2)}\right)^{j}\left(\bar{z}_{k}^{(2)}\right)^{l} \rho_{2}\left(z_{k}^{(2)}\right)}{\left(\sum_{k=1}^{N} z_{k}^{(2)} \bar{z}_{k}^{(2)} \rho_{2}\left(z_{k}^{(2)}\right)\right)^{\frac{j+l}{2}}}=\frac{\sum_{k=1}^{N}\left(\lambda z_{k}^{(1)}\right)^{j}\left(\lambda \bar{z}_{k}^{(1)}\right)^{l} \rho_{1}\left(z_{k}^{(1)}\right)}{\left(\sum_{k=1}^{N}\left(\lambda z_{k}^{(1)}\right)\left(\lambda \bar{z}_{k}^{(1)}\right) \rho_{1}\left(z_{k}^{(1)}\right)\right)^{\frac{j+l}{2}}} \\
= & \frac{\sum_{k=1}^{N} \lambda^{j+l}\left(z_{k}^{(1)}\right)^{j}\left(\bar{z}_{k}^{(1)}\right)^{l} \rho_{1}\left(z_{k}^{(1)}\right)}{\left(\sum_{k=1}^{N} \lambda^{2} z_{k}^{(1)} \bar{z}_{k}^{(1)} \rho_{1}\left(z_{k}^{(1)}\right)\right)^{\frac{j+l}{2}}}=\frac{\lambda^{j+l} \sum_{k=1}^{N}\left(z_{k}^{(1)}\right)^{j}\left(\bar{z}_{k}^{(1)}\right)^{l} \rho_{1}\left(z_{k}^{(1)}\right)}{\lambda^{j+l}\left(\sum_{k=1}^{N} z_{k}^{(1)} \bar{z}_{k}^{(1)} \rho_{1}\left(z_{k}^{(1)}\right)\right)^{\frac{j+l}{2}}} \\
= & \frac{\sum_{k=1}^{N}\left(z_{k}^{(1)}\right)^{j}\left(\bar{z}_{k}^{(1)}\right)^{l} \rho_{1}\left(z_{k}^{(1)}\right)}{\left(\sum_{k=1}^{N} z_{k}^{(1)} \bar{z}_{k}^{(1)} \rho_{1}\left(z_{k}^{(1)}\right)\right)^{\frac{j+l}{2}}}=\hat{\mu}_{j, l}^{(1)} .
\end{aligned}
$$

Therefore $T_{\text {scale }}^{r}\left(I_{1}\right)=T_{\text {scale }}^{r}\left(I_{2}\right)$ for any $r \in \mathbb{Z}_{\geq 0}$. i.e.

$\Leftarrow$ If $T_{\text {scale }}^{r}\left(I_{1}\right)=T_{\text {scale }}^{r}\left(I_{2}\right)$ for $r \geq 2 N-2$, from Corollary 1 , we conclude that $I_{1}^{\text {scale }}=I_{2}^{\text {scale }}$,

$$
\left\{\left(z_{k, \text { scale }}^{(1)}, \rho_{1}\left(z_{k, \text { scale }}^{(1)}\right)\right)\right\}_{k=1}^{N}=\left\{\left(z_{k, \text { scale }}^{(2)}, \rho_{2}\left(z_{k, \text { scale }}^{(2)}\right)\right)\right\}_{k=1}^{N} \text {. }
$$

Hence $\exists \lambda_{1}, \lambda_{2} \in \mathbb{R}_{+}$s.t. $z_{k, \text { scale }}^{(1)}=\lambda_{1} z_{k}^{(1)}, z_{k \text {,scale }}^{(2)}=\lambda_{2} z_{k}^{(2)}$ with $\rho_{1}\left(z_{k, \text { scale }}^{(1)}\right)=\rho_{1}\left(z_{k}^{(1)}\right)$ and $\rho_{2}\left(z_{k, \text { scale }}^{(2)}\right)=\rho_{2}\left(z_{k}^{(2)}\right)$ for any $k=1,2, \ldots, N$. After relabeling, we have $z_{k, \text { scale }}^{(1)}=z_{k, \text { scale }}^{(2)}$ and $\rho_{1}\left(z_{k, \text { scale }}^{(1)}\right)=\rho_{2}\left(z_{k, \text { scale }}^{(2)}\right)$. Then $\exists \frac{\lambda_{1}}{\lambda_{2}} \in \mathbb{R}_{+}=G$ satisfying

$$
z_{k}^{(1)} \frac{\lambda_{1}}{\lambda_{2}}=z_{k}^{(2)}, \quad \rho_{1}\left(z_{k}^{(1)} \frac{\lambda_{2}}{\lambda_{1}}\right)=\rho_{1}\left(z_{k}^{(1)}\right)=\rho_{2}\left(z_{k}^{(2)}\right),
$$

i.e. $\exists g=\frac{\lambda_{2}}{\lambda_{1}} \in G=\mathbb{R}_{+}$such that $g \circ I_{1}=I_{2}$.

More generally, consider the action of group $G$ of diagonal matrices on $\mathbb{R}_{+}^{2}$ by scaling

$$
\begin{aligned}
& \left(\left(\begin{array}{cc}
\lambda_{1} & 0 \\
0 & \lambda_{2}
\end{array}\right),\left(\begin{array}{l}
x \\
y
\end{array}\right)\right) \mapsto\left(\begin{array}{cc}
\lambda_{1} & 0 \\
0 & \lambda_{2}
\end{array}\right)\left(\begin{array}{l}
x \\
y
\end{array}\right)=\left(\begin{array}{l}
\lambda_{1} x \\
\lambda_{2} y
\end{array}\right), \\
& \forall\left(\begin{array}{cc}
\lambda_{1} & 0 \\
0 & \lambda_{2}
\end{array}\right) \in G, \quad \forall\left(\begin{array}{l}
x \\
y
\end{array}\right) \in \mathbb{R}^{2} .
\end{aligned}
$$

Then the induced transformation on the image $I=\left\{\left(z_{k}, \rho\left(z_{k}\right)\right)\right\}_{k=1}^{N}$ is

$$
\begin{aligned}
& \left(\begin{array}{cc}
\lambda_{1} & 0 \\
0 & \lambda_{2}
\end{array}\right) \circ\left\{\left(z_{k}, \rho\left(z_{k}\right)\right)\right\}_{k=1}^{N}=\left\{\left(\lambda_{1} x_{k}+i \lambda_{2} y_{k}, \rho\left(z_{k}\right)\right)\right\}_{k=1}^{N}, \\
& \forall\left(\begin{array}{cc}
\lambda_{1} & 0 \\
0 & \lambda_{2}
\end{array}\right) \in G, \quad z_{k}=x_{k}+i y_{k} .
\end{aligned}
$$

In other words, the image is scaled by a factor $\lambda_{1}$ horizontally and scaled by $\lambda_{2}$ vertically. 
Notice that after the transformation, the pixel coordinates become

$$
\begin{aligned}
& \hat{z}_{k}=\lambda_{1} x_{k}+i \lambda_{2} y_{k}=\lambda_{1} \frac{z_{k}+\bar{z}_{k}}{2}+i \lambda_{2} \frac{z_{k}-\bar{z}_{k}}{2 i}=\frac{\lambda_{1}+\lambda_{2}}{2} z_{k}+\frac{\lambda_{1}-\lambda_{2}}{2} \bar{z}_{k}, \\
& \overline{\hat{z}}_{k}=\lambda_{1} x_{k}-i \lambda_{2} y_{k}=\lambda_{1} \frac{z_{k}+\bar{z}_{k}}{2}-i \lambda_{2} \frac{z_{k}-\bar{z}_{k}}{2 i}=\frac{\lambda_{1}-\lambda_{2}}{2} z_{k}+\frac{\lambda_{1}+\lambda_{2}}{2} \bar{z}_{k} .
\end{aligned}
$$

Then we have the transformed complex moments

$$
\begin{aligned}
& \hat{\mu}_{j, l}=\sum_{k=1}^{N} \hat{z}_{k}^{j} \overline{\hat{z}}_{k}^{l} \rho\left(\hat{z}_{k}\right)=\sum_{k=1}^{N}\left(\frac{\lambda_{1}+\lambda_{2}}{2} z_{k}+\frac{\lambda_{1}-\lambda_{2}}{2} \bar{z}_{k}\right)^{j}\left(\frac{\lambda_{1}-\lambda_{2}}{2} z_{k}+\frac{\lambda_{1}+\lambda_{2}}{2} \bar{z}_{k}\right)^{l} \rho\left(z_{k}\right) \\
& =\sum_{k=1}^{N} \rho\left(z_{k}\right)\left[\sum_{s=0}^{j}\left(\begin{array}{l}
j \\
s
\end{array}\right)\left(\frac{\lambda_{1}+\lambda_{2}}{2}\right)^{s} z_{k}^{s}\left(\frac{\lambda_{1}-\lambda_{2}}{2}\right)^{j-s} \bar{z}_{k}^{j-s}\right] \\
& \times\left[\sum_{t=0}^{l}\left(\begin{array}{l}
l \\
t
\end{array}\right)\left(\frac{\lambda_{1}-\lambda_{2}}{2}\right)^{t} z_{k}^{t}\left(\frac{\lambda_{1}+\lambda_{2}}{2}\right)^{l-t} \bar{z}_{k}^{l-t}\right] \\
& =\sum_{k=1}^{N} \rho\left(z_{k}\right)\left[\sum_{s=0}^{j} \sum_{t=0}^{l}\left(\begin{array}{l}
j \\
s
\end{array}\right)\left(\begin{array}{l}
l \\
t
\end{array}\right)\left(\frac{\lambda_{1}+\lambda_{2}}{2}\right)^{l-t+s}\left(\frac{\lambda_{1}-\lambda_{2}}{2}\right)^{j-s+t} z_{k}^{s+t} \bar{z}_{k}^{j+l-s-t}\right] \\
& =\sum_{s=0}^{j} \sum_{t=0}^{l}\left(\begin{array}{l}
j \\
s
\end{array}\right)\left(\begin{array}{l}
l \\
t
\end{array}\right)\left(\frac{\lambda_{1}+\lambda_{2}}{2}\right)^{l-t+s}\left(\frac{\lambda_{1}-\lambda_{2}}{2}\right)^{j-s+t}\left[\sum_{k=1}^{N} \rho\left(z_{k}\right) z_{k}^{s+t} \bar{z}_{k}^{j+l-s-t}\right] \\
& =\frac{1}{2^{j+l}} \sum_{s=0}^{j} \sum_{t=0}^{l}\left(\begin{array}{l}
j \\
s
\end{array}\right)\left(\begin{array}{l}
l \\
t
\end{array}\right)\left(\lambda_{1}+\lambda_{2}\right)^{l-t+s}\left(\lambda_{1}-\lambda_{2}\right)^{j-s+t} \mu_{s+t, j+l-s-t}, \quad \forall j, l \in \mathbb{Z}_{\geq 0},
\end{aligned}
$$

which is a linear combination of the last row of the Pascal triangle $T^{j+l}(I)$.

Example 3. Consider the action of $G=\{z \in \mathbb{C}|| z \mid=1\}$ on $\mathbb{C}$ by rotation

$$
\left(e^{i \theta_{0}}, z\right) \mapsto z e^{i \theta_{0}}, \quad \forall e^{i \theta_{0}} \in G, \quad \forall z \in \mathbb{C} .
$$

Then the induced transformation on the image $I=\left\{\left(z_{k}, \rho\left(z_{k}\right)\right)\right\}_{k=1}^{N}$ is

$$
e^{i \theta_{0}} \circ\left\{\left(z_{k}, \rho\left(z_{k}\right)\right)\right\}_{k=1}^{N}=\left\{\left(e^{i \theta_{0}} z_{k}, \rho\left(z_{k}\right)\right)\right\}_{k=1}^{N}, \quad \forall e^{i \theta_{0}} \in G .
$$

In other words, the image is rotated counterclockwise with an angle $\theta_{0}$. The transformed complex moments are

$$
\begin{aligned}
\mu_{j, l}^{\prime} & =\sum_{k=1}^{N} z_{k}^{\prime j} \bar{z}_{k}^{l} \rho\left(z_{k}^{\prime}\right)=\sum_{k=1}^{N}\left(z_{k} e^{i \theta_{0}}\right)^{j}\left(\bar{z}_{k} e^{-i \theta_{0}}\right)^{l} \rho\left(z_{k}\right)=\sum_{k=1}^{N} e^{i(j-l) \theta_{0}} z_{k}^{j} \bar{z}_{k}^{l} \rho\left(z_{k}\right) \\
& =e^{i(j-l) \theta_{0}} \sum_{k=1}^{N} z_{k}^{j} \bar{z}_{k}^{l} \rho\left(z_{k}\right)=\mu_{j, l} e^{i(j-l) \theta_{0}}, \quad \forall j, l \in \mathbb{Z}_{\geq 0} .
\end{aligned}
$$

Written in matrix form, the moment matrix for the new image $I^{\prime}$ after rotation is

$$
\tau_{N}\left(I^{\prime}\right)=\left(\begin{array}{ccccc}
1 & 0 & 0 & \cdots & 0 \\
0 & e^{-i \theta_{0}} & 0 & \cdots & 0 \\
0 & 0 & e^{-i 2 \theta_{0}} & \cdots & 0 \\
\vdots & \vdots & \vdots & \ddots & \vdots \\
0 & \cdots & \cdots & 0 & e^{-i(N-1) \theta_{0}}
\end{array}\right) \tau_{N}(I)\left(\begin{array}{ccccc}
1 & 0 & 0 & \cdots & 0 \\
0 & e^{i \theta_{0}} & 0 & \cdots & 0 \\
0 & 0 & e^{i 2 \theta_{0}} & \cdots & 0 \\
\vdots & \vdots & \vdots & \ddots & \vdots \\
0 & \cdots & \cdots & 0 & e^{i(N-1) \theta_{0}}
\end{array}\right) .
$$


Now we will use the moving frame method of Fels and Olver to obtain a set of invariant functions of the moments. If $\mu_{0,2} \neq 0$, we normalize the imaginary part of $\mu_{0,2}^{\prime}$ to zero by specifying the rotation angle $\theta_{0}$. Since $\mu_{0,2}^{\prime}=\mu_{0,2} e^{-i 2 \theta_{0}}$, looking at $\vec{\mu}_{0,2}$ as a vector in $\mathbb{R}^{2}$ representing the complex number $\mu_{0,2}$, we set

$$
2 \theta_{0}=\varangle\left(\vec{\mu}_{0,2}, \vec{e}_{1}\right)+2 k \pi, \quad k \in \mathbb{Z} .
$$

Here $\varangle(\vec{x}, \vec{y})=\tan ^{-1}\left(\frac{y_{2}}{y_{1}}\right)-\tan ^{-1}\left(\frac{x_{2}}{x_{1}}\right) \in(-\pi, \pi]$ denotes the angle from $\vec{x}$ to $\vec{y}, \vec{e}_{1}=(1,0)^{T}$ is one of the standard basis of $\mathbb{R}^{2}$. The real part of $\mu_{0,2}^{\prime}$ then reduces to its magnitude $\left|\mu_{0,2}\right|$.

Since $\theta_{0} \in(-\pi, \pi], 2 \theta_{0} \in(-2 \pi, 2 \pi]$. To uniquely determine the value of $\theta_{0}$, we consider $\mu_{1,2}^{\prime}=\mu_{1,2} e^{-i \theta_{0}}$. We choose $\theta_{0}$ such that $\operatorname{Re}\left(\mu_{1,2}^{\prime}\right) \geq 0$, which leads to the moving frame formulae

$$
\theta_{0}= \begin{cases}\frac{1}{2} \varangle\left(\vec{\mu}_{0,2}, \vec{e}_{1}\right) & \text { if } \varangle\left(\vec{\mu}_{1,2}, \vec{e}_{1}\right)-\frac{1}{2} \varangle\left(\vec{\mu}_{0,2}, \vec{e}_{1}\right) \in\left[-\frac{\pi}{2}, \frac{\pi}{2}\right], \\ \frac{1}{2} \varangle\left(\vec{\mu}_{0,2}, \vec{e}_{1}\right)+\pi & \text { if } \varangle\left(\vec{\mu}_{1,2}, \vec{e}_{1}\right)-\frac{1}{2} \varangle\left(\vec{\mu}_{0,2}, \vec{e}_{1}\right) \in\left(\frac{\pi}{2}, \frac{3 \pi}{2}\right], \\ \frac{1}{2} \varangle\left(\vec{\mu}_{0,2}, \vec{e}_{1}\right)-\pi & \text { if } \varangle\left(\vec{\mu}_{1,2}, \vec{e}_{1}\right)-\frac{1}{2} \varangle\left(\vec{\mu}_{0,2}, \vec{e}_{1}\right) \in\left[-\frac{3 \pi}{2},-\frac{\pi}{2}\right) .\end{cases}
$$

By applying the moving frame to the moment matrix, we obtain the matrix

$$
\tau_{N}^{\prime}(I)=\left(\begin{array}{ccccc}
1 & 0 & 0 & \cdots & 0 \\
0 & e^{-i \theta_{0}} & 0 & \cdots & 0 \\
0 & 0 & e^{-i 2 \theta_{0}} & \cdots & 0 \\
\vdots & \vdots & \vdots & \ddots & \vdots \\
0 & \cdots & \cdots & 0 & e^{-i(N-1) \theta_{0}}
\end{array}\right) \tau_{N}(I)\left(\begin{array}{ccccc}
1 & 0 & 0 & \cdots & 0 \\
0 & e^{i \theta_{0}} & 0 & \cdots & 0 \\
0 & 0 & e^{i 2 \theta_{0}} & \cdots & 0 \\
\vdots & \vdots & \vdots & \ddots & \vdots \\
0 & \cdots & \cdots & 0 & e^{i(N-1) \theta_{0}}
\end{array}\right),
$$

with $\theta_{0}$ satisfying (11). By equivariance of the moving frame, all the entries of $\tau_{N}^{\prime}(I)$ are invariant under rotation.

By normalizing the coordinates of $T^{r}(I)$, we obtain the rotational invariant Pascal triangle $T_{\text {rotate }}^{r}(I)$ for a discrete image $I$ :

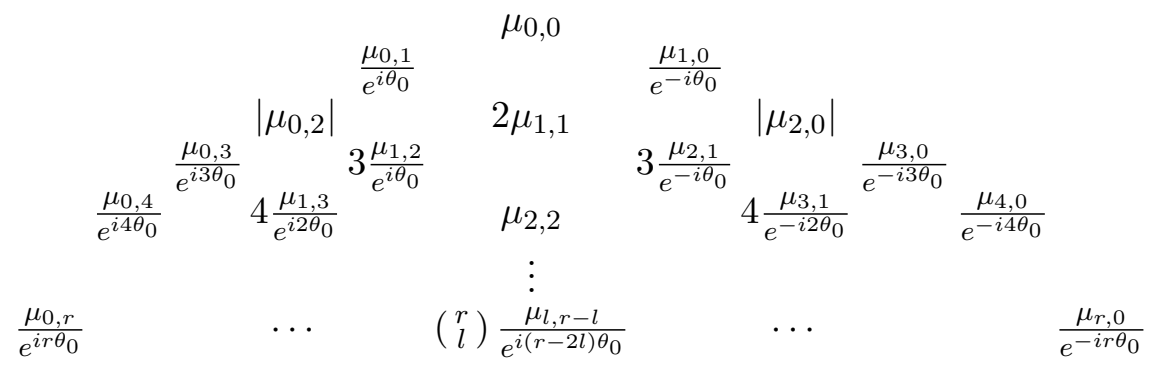

Observe that the corresponding $n$-th order moment $m_{n}^{\prime}(\theta)=m_{n}\left(\theta-\theta_{0}\right)$, with $\theta_{0}$ defined as in (11), is invariant under rotations.

Lemma 7 (orbit separation property of $T_{\text {rotate }}^{2 N-2}(I)$ ). Let $I_{1}, I_{2}$ be two discrete gray scale images with the same number $N$ of pixels. There exists a rotation $g \in\{z \in \mathbb{C}|| z \mid=1\}$ such that $g \circ I_{1}=I_{2}$, where $\circ$ is defined as in $(10) \Longleftrightarrow T_{\text {rotate }}^{r}\left(I_{1}\right)=T_{\text {rotate }}^{r}\left(I_{2}\right)$ for $r \geq 2 N-2$.

Proof. $\Rightarrow$ If $\exists g \in G$ such that $g \circ I_{1}=I_{2}$, we have $z_{k}^{(2)}=z_{k}^{(1)} e^{i \theta_{0}}$ and $\rho_{1}\left(z_{k}^{(1)}\right)=\rho_{2}\left(z_{k}^{(2)}\right)$, for some $e^{i \theta_{0}} \in\{z \in \mathbb{C}|| z \mid=1\}, k=1, \ldots, N$. For $I_{1}$ and $I_{2}$, the corresponding scaling invariant moments are

$$
\hat{\mu}_{j, l}^{(1)}=\mu_{j, l}^{(1)} e^{-i(l-j) \theta_{1}}=\sum_{k=1}^{N}\left(z_{k}^{(1)}\right)^{j}\left(\bar{z}_{k}^{(1)}\right)^{l} \rho_{1}\left(z_{k}^{(1)}\right) e^{i(j-l) \theta_{1}},
$$




$$
\begin{aligned}
\hat{\mu}_{j, l}^{(2)} & =\mu_{j, l}^{(2)} e^{-i(l-j) \theta_{2}}=\sum_{k=1}^{N}\left(z_{k}^{(2)}\right)^{j}\left(\bar{z}_{k}^{(2)}\right)^{l} \rho_{2}\left(z_{k}^{(2)}\right) e^{i(j-l) \theta_{2}} \\
& =\sum_{k=1}^{N}\left(z_{k}^{(1)} e^{i \theta_{0}}\right)^{j}\left(\bar{z}_{k}^{(1)} e^{-i \theta_{0}}\right)^{l} \rho_{1}\left(z_{k}^{(1)}\right) e^{i(j-l) \theta_{2}}=\sum_{k=1}^{N}\left(z_{k}^{(1)}\right)^{j}\left(\bar{z}_{k}^{(1)}\right)^{l} \rho_{1}\left(z_{k}^{(1)}\right) e^{i(j-l)\left(\theta_{0}+\theta_{2}\right)} .
\end{aligned}
$$

Since $\mu_{0,2}^{(2)}=\mu_{0,2}^{(1)} e^{-i 2 \theta_{0}}, \mu_{1,2}^{(2)}=\mu_{1,2}^{(1)} e^{-i \theta_{0}}$, we have

$$
\begin{aligned}
& \varangle\left(\vec{\mu}_{0,2}^{(2)}, \vec{e}_{1}\right)=\varangle\left(\vec{\mu}_{0,2}^{(1)}, \vec{e}_{1}\right)-2 \theta_{0}+2 k_{1} \pi, \\
& \varangle\left(\vec{\mu}_{1,2}^{(2)}, \vec{e}_{1}\right)=\varangle\left(\vec{\mu}_{1,2}^{(1)}, \vec{e}_{1}\right)-\theta_{0}+2 k_{2} \pi, \quad k_{1}, k_{2} \in \mathbb{Z} .
\end{aligned}
$$

Notice that $\varangle\left(\vec{\mu}_{0,2}^{(l)}, \vec{e}_{1}\right), \varangle\left(\vec{\mu}_{1,2}^{(l)}, \vec{e}_{1}\right), \theta_{0} \in(-\pi, \pi]$, for $l=1,2$. Hence $k_{1}, k_{2}=0, \pm 1$. To decide $\theta_{1}$ and $\theta_{2}$, we consider

$$
\varangle\left(\vec{\mu}_{1,2}^{(2)}, \vec{e}_{1}\right)-\frac{1}{2} \varangle\left(\vec{\mu}_{0,2}^{(2)}, \vec{e}_{1}\right)=\varangle\left(\vec{\mu}_{1,2}^{(1)}, \vec{e}_{1}\right)-\frac{1}{2} \varangle\left(\vec{\mu}_{0,2}^{(1)}, \vec{e}_{1}\right)+\left(2 k_{2}-k_{1}\right) \pi
$$

by using (12).

If $\varangle\left(\vec{\mu}_{1,2}^{(2)}, \vec{e}_{1}\right)-\frac{1}{2} \varangle\left(\vec{\mu}_{0,2}^{(2)}, \vec{e}_{1}\right) \in\left[-\frac{\pi}{2}, \frac{\pi}{2}\right]$, from (11) we know that $\theta_{2}=\frac{1}{2} \varangle\left(\vec{\mu}_{0,2}^{(2)}, \vec{e}_{1}\right)$. Since $\varangle\left(\vec{\mu}_{1,2}^{(1)}, \vec{e}_{1}\right)-\frac{1}{2} \varangle\left(\vec{\mu}_{0,2}^{(1)}, \vec{e}_{1}\right) \in\left[-\frac{3 \pi}{2}, \frac{3 \pi}{2}\right]$, then $2 k_{2}-k_{1}$ is either 0 or \pm 1 in (13). If $k_{1}=0, k_{2}=0$, there is $\varangle\left(\vec{\mu}_{1,2}^{(1)}, \vec{e}_{1}\right)-\frac{1}{2} \varangle\left(\vec{\mu}_{0,2}^{(1)}, \vec{e}_{1}\right) \in\left[-\frac{\pi}{2}, \frac{\pi}{2}\right]$. Hence $\theta_{1}=\frac{1}{2} \varangle\left(\vec{\mu}_{0,2}^{(1)}, \vec{e}_{1}\right)$. Then from (12) we have $\theta_{2}=\theta_{1}-\theta_{0}$. Thus $\hat{\mu}_{j, l}^{(2)}=\sum_{k=1}^{N}\left(z_{k}^{(1)}\right)^{j}\left(\bar{z}_{k}^{(1)}\right)^{l} \rho_{1}\left(z_{k}^{(1)}\right) e^{i(j-l) \theta_{1}}=\hat{\mu}_{j, l}^{(1)}$. If $k_{1}=1, k_{2}=0$, there is $\varangle\left(\vec{\mu}_{1,2}^{(1)}, \vec{e}_{1}\right)-\frac{1}{2} \varangle\left(\vec{\mu}_{0,2}^{(1)}, \vec{e}_{1}\right) \in\left(\frac{\pi}{2}, \frac{3 \pi}{2}\right]$. Hence $\theta_{1}=\frac{1}{2} \varangle\left(\vec{\mu}_{0,2}^{(1)}, \vec{e}_{1}\right)+\pi$. Then from (12) we still have $\theta_{2}=\theta_{1}-\theta_{0}$. Thus $\hat{\mu}_{j, l}^{(2)}=\hat{\mu}_{j, l}^{(1)}$. If $k_{1}=1, k_{2}=1$, there is $\varangle\left(\vec{\mu}_{1,2}^{(1)}, \vec{e}_{1}\right)-\frac{1}{2} \varangle\left(\vec{\mu}_{0,2}^{(1)}, \vec{e}_{1}\right) \in$ $\left[-\frac{3 \pi}{2},-\frac{\pi}{2}\right)$. Hence $\theta_{1}=\frac{1}{2} \varangle\left(\vec{\mu}_{0,2}^{(1)}, \vec{e}_{1}\right)-\pi$. Then from (12) we have $\theta_{2}=\theta_{1}-\theta_{0}+2 \pi$. Thus $\hat{\mu}_{j, l}^{(2)}=\sum_{k=1}^{N}\left(z_{k}^{(1)}\right)^{j}\left(\bar{z}_{k}^{(1)}\right)^{l} \rho_{1}\left(z_{k}^{(1)}\right) e^{i(j-l) \theta_{1}}=\hat{\mu}_{j, l}^{(1)}$.

If $\varangle\left(\vec{\mu}_{1,2}^{(2)}, \vec{e}_{1}\right)-\frac{1}{2} \varangle\left(\vec{\mu}_{0,2}^{(2)}, \vec{e}_{1}\right) \in\left(\frac{\pi}{2}, \frac{3 \pi}{2}\right]$, from (11) we know that $\theta_{2}=\frac{1}{2} \varangle\left(\vec{\mu}_{0,2}^{(2)}, \vec{e}_{1}\right)+\pi$. Since $\varangle\left(\vec{\mu}_{1,2}^{(1)}, \vec{e}_{1}\right)-\frac{1}{2} \varangle\left(\vec{\mu}_{0,2}^{(1)}, \vec{e}_{1}\right) \in\left[-\frac{3 \pi}{2}, \frac{3 \pi}{2}\right]$, then $2 k_{2}-k_{1}=0,1,2$ in (13). If $k_{1}=1, k_{2}=1$, there is $\varangle\left(\vec{\mu}_{1,2}^{(1)}, \vec{e}_{1}\right)-\frac{1}{2} \varangle\left(\vec{\mu}_{0,2}^{(1)}, \vec{e}_{1}\right) \in\left[-\frac{\pi}{2}, \frac{\pi}{2}\right]$. Hence $\theta_{1}=\frac{1}{2} \varangle\left(\vec{\mu}_{0,2}^{(1)}, \vec{e}_{1}\right)$. Then from (12) we have $\theta_{2}-\pi=\theta_{1}-\theta_{0}+\pi$. Thus $\hat{\mu}_{j, l}^{(2)}=\sum_{k=1}^{N}\left(z_{k}^{(1)}\right)^{j}\left(\bar{z}_{k}^{(1)}\right)^{l} \rho_{1}\left(z_{k}^{(1)}\right) e^{i(j-l) \theta_{1}}=\hat{\mu}_{j, l}^{(1)}$. If $k_{1}=0$, $k_{2}=0$, there is $\varangle\left(\vec{\mu}_{1,2}^{(1)}, \vec{e}_{1}\right)-\frac{1}{2} \varangle\left(\vec{\mu}_{0,2}^{(1)}, \vec{e}_{1}\right) \in\left(\frac{\pi}{2}, \frac{3 \pi}{2}\right]$. Hence $\theta_{1}=\frac{1}{2} \varangle\left(\vec{\mu}_{0,2}^{(1)}, \vec{e}_{1}\right)+\pi$. Then from (12) we have $\theta_{2}-\pi=\theta_{1}-\theta_{0}-\pi$. Thus $\hat{\mu}_{j, l}^{(2)}=\hat{\mu}_{j, l}^{(1)}$. If $k_{1}=0, k_{2}=1$, there is $\varangle\left(\vec{\mu}_{1,2}^{(1)}, \vec{e}_{1}\right)-\frac{1}{2} \varangle\left(\vec{\mu}_{0,2}^{(1)}, \vec{e}_{1}\right) \in\left[-\frac{3 \pi}{2},-\frac{\pi}{2}\right)$. Hence $\theta_{1}=\frac{1}{2} \varangle\left(\vec{\mu}_{0,2}^{(1)}, \vec{e}_{1}\right)-\pi$. Then from (12) we have $\theta_{2}-\pi=\theta_{1}-\theta_{0}+\pi$. Thus $\hat{\mu}_{j, l}^{(2)}=\sum_{k=1}^{N}\left(z_{k}^{(1)}\right)^{j}\left(\bar{z}_{k}^{(1)}\right)^{l} \rho_{1}\left(z_{k}^{(1)}\right) e^{i(j-l) \theta_{1}}=\hat{\mu}_{j, l}^{(1)}$.

If $\varangle\left(\vec{\mu}_{1,2}^{(2)}, \vec{e}_{1}\right)-\frac{1}{2} \varangle\left(\vec{\mu}_{0,2}^{(2)}, \vec{e}_{1}\right) \in\left[-\frac{3 \pi}{2},-\frac{\pi}{2}\right)$, through the similar discussion, we can still conclude that $\hat{\mu}_{j, l}^{(2)}=\hat{\mu}_{j, l}^{(1)}$. Therefore $T_{\text {scale }}^{r}\left(I_{1}\right)=T_{\text {scale }}^{r}\left(I_{2}\right)$ for any $r \in \mathbb{Z}_{\geq 0}$.

$\Leftarrow$ If $T_{\text {rotate }}^{r}\left(I_{1}\right)=T_{\text {rotate }}^{r}\left(I_{2}\right)$ for $r \geq 2 N-2$, from Corollary 1 , we conclude that $I_{1}^{\text {rotate }}=$ $I_{2}^{\text {rotate }}$, i.e.

$$
\left\{\left(z_{k, \text { rotate }}^{(1)}, \rho_{1}\left(z_{k, \text { rotate }}^{(1)}\right)\right)\right\}_{k=1}^{N}=\left\{\left(z_{k, \text { rotate }}^{(2)}, \rho_{2}\left(z_{k, \text { rotate }}^{(2)}\right)\right)\right\}_{k=1}^{N} \text {. }
$$

Hence $\exists e^{i \theta_{1}}, e^{i \theta_{2}} \in\{z \in \mathbb{C}|| z \mid=1\}$ s.t. $z_{k \text {,rotate }}^{(1)}=z_{k}^{(1)} e^{i \theta_{1}}, z_{k, \text { rotate }}^{(2)}=z_{k}^{(2)} e^{i \theta_{2}}$ with $\rho_{1}\left(z_{k, \text { rotate }}^{(1)}\right)=$ $\rho_{1}\left(z_{k}^{(1)}\right)$ and $\rho_{2}\left(z_{k, \text { rotate }}^{(2)}\right)=\rho_{2}\left(z_{k}^{(2)}\right)$ for any $k=1,2, \ldots, N$. Without loss of generality, we 
assume $z_{k, \text { rotate }}^{(1)}=z_{k, \text { rotate }}^{(2)}$ and $\rho_{1}\left(z_{k, \text { rotate }}^{(1)}\right)=\rho_{2}\left(z_{k, \text { rotate }}^{(2)}\right)$. Then $\exists e^{i\left(\theta_{1}-\theta_{2}\right)} \in\{z \in \mathbb{C}|| z \mid=1\}=$ $G$ satisfying

$$
z_{k}^{(1)} e^{i\left(\theta_{1}-\theta_{2}\right)}=z_{k}^{(2)}, \quad \rho_{1}\left(z_{k}^{(1)} e^{i\left(\theta_{1}-\theta_{2}\right)}\right)=\rho_{1}\left(z_{k}^{(1)}\right)=\rho_{2}\left(z_{k}^{(2)}\right),
$$

i.e. $\exists g=e^{i\left(\theta_{1}-\theta_{2}\right)} \in G=\{z \in \mathbb{C}|| z \mid=1\}$ such that $g \circ I_{1}=I_{2}$.

In conclusion, we have the translation, scaling and rotation invariant Pascal triangle $T_{\text {int }}^{r}(I)$ :

$$
\begin{aligned}
& \begin{array}{ccc} 
& \tilde{\mu}_{0,0} e^{-i \theta_{0}} \\
\sqrt{\tilde{\mu}_{1,1}} & \frac{\tilde{\mu}_{1,0} e^{i \theta_{0}}}{\sqrt{\tilde{\mu}_{1,1}}}
\end{array} \\
& \frac{\tilde{\mu}_{0,3} e^{-i 3 \theta_{0}}}{\tilde{\mu}_{1,1}^{3 / 2}} \stackrel{\frac{\left|\tilde{\mu}_{0,2}\right|}{\tilde{\mu}_{1,1}}}{3} \frac{\tilde{\mu}_{1,2} e^{-i \theta_{0}}}{\tilde{\mu}_{1,1}^{3 / 2}} \\
& 2 \\
& 3 \frac{\tilde{\mu}_{2,1} e^{i \theta_{0}}}{\tilde{\mu}_{1,1}^{3 / 2}} \quad \frac{\frac{\left|\tilde{\mu}_{2,0}\right|}{\tilde{\mu}_{1,1}}}{\frac{\tilde{\mu}_{3,0} e^{i 3 \theta_{0}}}{\tilde{\mu}_{1,1}^{3 / 2}}} \\
& \begin{array}{lllll}
\frac{\tilde{\mu}_{0, r} e^{-i r \theta_{0}}}{\tilde{\mu}_{1,1}^{r / 2}} & \ldots & \frac{\left(\begin{array}{c}
r \\
l
\end{array}\right) \tilde{\mu}_{l, r-l}}{\tilde{\mu}_{1,1}^{r / 2} e^{i(r-2 l) \theta_{0}}} & \ldots & \frac{\tilde{\mu}_{r, 0} e^{i r \theta_{0}}}{\tilde{\mu}_{1,1}^{r / 2}}
\end{array}
\end{aligned}
$$

\section{Geometric interpretation of the moments}

\subsection{Shape elongation}

Intuitively, the elongation of a shape is described by the relationship between its length and its width. It is thus a property that is invariant under translation, scaling and rotation of the image. To characterize the shape elongation, we therefore consider the invariantization $\hat{\tilde{m}}_{2}^{\prime}(\theta)$ of the second order moment $m_{2}(\theta)$ with respect to translation, scaling and rotation. By combining the results of Examples 1, 2 and 3, we have

$$
\begin{aligned}
\hat{\tilde{m}}_{2}^{\prime}(\theta) & =\frac{1}{4}\left(\frac{\left|\tilde{\mu}_{0,2}\right|}{\tilde{\mu}_{1,1}} e^{i 2 \theta}+2+\frac{\left|\tilde{\mu}_{2,0}\right|}{\tilde{\mu}_{1,1}} e^{-i 2 \theta}\right) \\
& =\frac{1}{4}\left(\frac{\left|\tilde{\mu}_{0,2}\right|}{\tilde{\mu}_{1,1}}(\cos (2 \theta)+i \sin (2 \theta))+2+\frac{\left|\tilde{\mu}_{2,0}\right|}{\tilde{\mu}_{1,1}}(\cos (2 \theta)-i \sin (2 \theta))\right) \\
& =\frac{1}{4}\left(2 \frac{\left|\tilde{\mu}_{0,2}\right|}{\tilde{\mu}_{1,1}} \cos (2 \theta)+2\right)=\frac{1}{2}\left(\frac{\left|\tilde{\mu}_{0,2}\right|}{\tilde{\mu}_{1,1}} \cos (2 \theta)+1\right),
\end{aligned}
$$

where

$$
\tilde{\mu}_{1,1}=\sum_{k=1}^{N}\left(z_{k}-z_{0}\right)\left(\bar{z}_{k}-\bar{z}_{0}\right) \rho\left(z_{k}\right)=\sum_{k=1}^{N}\left(\left(x_{k}-x_{0}\right)^{2}+\left(y_{k}-y_{0}\right)^{2}\right) \rho\left(z_{k}\right)>0 .
$$

Recall that when making the moments scaling invariant, we normalized $\hat{\tilde{\mu}}_{1,1}$ to 1 by dividing each $\tilde{\mu}_{j, l}$ by the corresponding power of $\sqrt{\tilde{\mu}_{1,1}}$. Hence $\sqrt{\tilde{\mu}_{1,1}}$ represents the scale of the image.

Equation (14) indicates that the quantity $\frac{\left|\tilde{\mu}_{0,2}\right|}{\tilde{\mu}_{1,1}}$ prescribes the relationship between the maximum and the minimum values of the standard deviation of the random transform. It thus gives us a quantification of the elongation of the shape illustrated by the image ${ }^{5}$.

Lemma 8. If not all $\rho\left(z_{k}\right)$ are zero, then $0 \leq \frac{\left|\tilde{\mu}_{0,2}\right|}{\tilde{\mu}_{1,1}} \leq 1$.

\footnotetext{
${ }^{5}$ A.R. Rostampour et al. [8] previously introduced the $\frac{n_{04}}{n_{02}^{2}}$ as a measure of elongation of the projections of an image. We observe that our measure of elongation has a lower order, and that it can be obtained without normalizing the angle of the image.
} 
Proof. By definition, $\left|\tilde{\mu}_{0,2}\right| \geq 0$ and $\tilde{\mu}_{1,1}>0$, hence $\frac{\left|\tilde{\mu}_{0,2}\right|}{\tilde{\mu}_{1,1}} \geq 0$. If $\frac{\left|\tilde{\mu}_{0,2}\right|}{\tilde{\mu}_{1,1}}>1$, since $\hat{\tilde{m}}_{2}^{\prime}(\theta)=$ $\frac{1}{2}\left(\frac{\left|\tilde{\mu}_{0,2}\right|}{\tilde{\mu}_{1,1}} \cos (2 \theta)+1\right)$, and $\hat{\tilde{m}}_{2}^{\prime}(\theta) \geq 0$ by definition, if we choose $\theta=\frac{\pi}{2}$, then $\frac{\left|\tilde{\mu}_{0,2}\right|}{\tilde{\mu}_{1,1}} \cos (2 \theta)+1<0$, which is a contradiction. Therefore $\frac{\left|\tilde{\mu}_{0,2}\right|}{\tilde{\mu}_{1,1}} \leq 1$.

The case $\frac{\left|\tilde{\mu}_{0,2}\right|}{\tilde{\mu}_{1,1}}=1$ corresponds to the most extreme elongation, namely the straight lines.

Lemma 9. The pixel coordinates $z_{k}$ lie on a single straight line if and only if $\frac{\left|\tilde{\mu}_{0,2}\right|}{\tilde{\mu}_{1,1}}=1$.

Proof. $\Rightarrow$ Suppose we have a straight line. As the line is put vertically, the projection of the line is a dot. Hence the second moment of the Radon transform is zero at that angle $\theta^{*}$, i.e.

$$
\hat{\tilde{m}}_{2}^{\prime}\left(\theta^{*}\right)=\frac{1}{2}\left(\frac{\left|\tilde{\mu}_{0,2}\right|}{\tilde{\mu}_{1,1}} \cos \left(2 \theta^{*}\right)+1\right)=0 .
$$

Since $-1 \leq \cos \left(2 \theta^{*}\right) \leq 1$ and from Lemma 8 we know that $0 \leq \frac{\left|\tilde{\mu}_{0,2}\right|}{\tilde{\mu}_{1,1}} \leq 1$ for any image, we can conclude that (15) is true only when $\frac{\left|\tilde{\mu}_{0,2}\right|}{\tilde{\mu}_{1,1}}=1$ and $\cos \left(2 \theta^{*}\right)=-1$. Hence $\frac{\left|\tilde{\mu}_{0,2}\right|}{\tilde{\mu}_{1,1}}=1$ is true.

$\Leftarrow$ Now suppose $\frac{\left|\tilde{\mu}_{0,2}\right|}{\tilde{\mu}_{1,1}}=1$. Combine the results in Examples 1, 2 and 3 , we conclude that

$$
\hat{\tilde{m}}_{2}^{\prime}(\theta)=\tilde{m}_{2}\left(\theta-\theta_{0}\right) / \tilde{\mu}_{1,1}=\frac{1}{2}\left(\frac{\left|\tilde{\mu}_{0,2}\right|}{\tilde{\mu}_{1,1}} \cos (2 \theta)+1\right), \quad \forall \theta \in\left(-\frac{\pi}{2}, \frac{\pi}{2}\right]
$$

where $\theta_{0}$ satisfies $(11)$. Since $\hat{\tilde{m}}_{n}^{\prime}\left(\frac{\pi}{2}\right)=0$, then there is $\tilde{\theta}=\frac{\pi}{2}-\theta_{0}$ such that at this angle the centralized second order moment $\tilde{m}_{2}(\tilde{\theta})$ of the image is zero.

Let the Radon transform $f_{\tilde{\theta}}(r)$ at angle $\tilde{\theta}$ be a discrete function. Without loss of generality, we assume that $\sum_{k=1}^{N} f_{\tilde{\theta}}\left(r_{k}(\theta)\right)=1$ and $f_{\tilde{\theta}}\left(r_{k}(\theta)\right) \geq 0$. Since

$$
\tilde{m}_{2}(\tilde{\theta})=\sum_{k=1}^{N}\left(r_{k}(\tilde{\theta})-r_{0}(\tilde{\theta})\right)^{2} f_{\tilde{\theta}}\left(r_{k}(\theta)\right)=0
$$

where $r_{0}(\tilde{\theta})$ is the projection of the centroid of the image, we observe that $f_{\tilde{\theta}}(r)=\delta\left(r-r_{0}(\tilde{\theta})\right)$. Then we conclude that the image lies on a line through $r_{0}(\tilde{\theta})$ with angle $\tilde{\theta}+\frac{\pi}{2}$ to the $x$-axis.

The other extreme case is when $\frac{\left|\tilde{\mu}_{0,2}\right|}{\tilde{\mu}_{1,1}}=0$. This corresponds to $\hat{\tilde{m}}_{2}^{\prime}(\theta)=$ const. So the standard deviation of the projection is the same for all directions. There are many ways for this to happen. One interesting case is the discrete analogue of rotation symmetries.

Definition 2. An object is said to have $\tilde{N}$-fold rotation symmetry ( $\tilde{N}$-FRS) if it is unchanged by a rotation around its centroid by $\frac{2 k \pi}{\tilde{N}}$, for all $k=1, \ldots, \tilde{N}$.

Lemma 10. If the image I has an $\tilde{N}-F R S$ with $\tilde{N}>2$, then $\frac{\left|\tilde{\mu}_{0,2}\right|}{\tilde{\mu}_{1,1}}=0$.

Proof. Suppose the data have $\tilde{N}$-FRS. For a certain point with distance $r_{k}, k=1,2, \ldots, M$, from the centroid, angle $\theta_{k}$ with $x$-axis and weight $\rho\left(z_{k}\right)$, there are $\tilde{N}_{k}-1$ more points with the same distance from the centroid and the same weight $\rho\left(z_{k}\right)$ but having angle $\theta_{k}+\frac{2 j \pi}{\tilde{N}_{k}}$, $j=1, \ldots, \tilde{N}_{k}-1$ with $x$-axis respectively. Then the moment $\tilde{\mu}_{0,2}$ can be written as

$$
\tilde{\mu}_{0,2}=\sum_{k=1}^{M} \rho\left(z_{k}\right)\left(\sum_{j=0}^{\tilde{N}_{k}-1}\left(r_{k} \cos \left(\theta_{k}+\frac{2 j \pi}{\tilde{N}_{k}}\right)-i r_{k} \sin \left(\theta_{k}+\frac{2 j \pi}{\tilde{N}_{k}}\right)\right)^{2}\right)
$$




$$
\begin{aligned}
& =\sum_{k=1}^{M} \rho\left(z_{k}\right)\left(\sum_{j=0}^{\tilde{N}_{k}-1} r_{k}^{2} \cos \left(2 \theta_{k}+\frac{4 j \pi}{\tilde{N}_{k}}\right)\right)-i \sum_{k=1}^{M} \rho\left(z_{k}\right)\left(\sum_{j=0}^{\tilde{N}_{k}-1} r_{k}^{2} \sin \left(2 \theta_{k}+\frac{4 j \pi}{\tilde{N}_{k}}\right)\right) \\
& =\sum_{k=1}^{M} \rho\left(z_{k}\right)\left(\sum_{j=0}^{\tilde{N}_{k}-1} r_{k}^{2} \cos \left(2 \theta_{k}\right) \cos \left(\frac{4 j \pi}{\tilde{N}_{k}}\right)-r_{k}^{2} \sin \left(2 \theta_{k}\right) \sin \left(\frac{4 j \pi}{\tilde{N}_{k}}\right)\right) \\
& -i \sum_{k=1}^{M} \rho\left(z_{k}\right)\left(\sum_{j=0}^{\tilde{N}_{k}-1} r_{k}^{2} \sin \left(2 \theta_{k}\right) \cos \left(\frac{4 j \pi}{\tilde{N}_{k}}\right)+r_{k}^{2} \cos \left(2 \theta_{k}\right) \sin \left(\frac{4 j \pi}{\tilde{N}_{k}}\right)\right) \\
& =\sum_{k=1}^{M} \rho\left(z_{k}\right)\left(r_{k}^{2} \cos \left(2 \theta_{k}\right)\left(\sum_{j=0}^{\tilde{N}_{k}-1} \cos \left(\frac{4 j \pi}{\tilde{N}_{k}}\right)\right)-r_{k}^{2} \sin \left(2 \theta_{k}\right)\left(\sum_{j=0}^{\tilde{N}_{k}-1} \sin \left(\frac{4 j \pi}{\tilde{N}_{k}}\right)\right)\right) \\
& -i \sum_{k=1}^{M} \rho\left(z_{k}\right)\left(r_{k}^{2} \sin \left(2 \theta_{k}\right)\left(\sum_{j=0}^{\tilde{N}_{k}-1} \cos \left(\frac{4 j \pi}{\tilde{N}_{k}}\right)\right)+r_{k}^{2} \cos \left(2 \theta_{k}\right)\left(\sum_{j=0}^{\tilde{N}_{k}-1} \sin \left(\frac{4 j \pi}{\tilde{N}_{k}}\right)\right)\right) \text {. }
\end{aligned}
$$

It can be shown that

$$
\sum_{j=0}^{\tilde{N}_{k}-1} \cos \left(\frac{4 j \pi}{\tilde{N}_{k}}\right)=0, \quad \sum_{j=0}^{\tilde{N}_{k}-1} \sin \left(\frac{4 j \pi}{\tilde{N}_{k}}\right)=0, \quad \forall \tilde{N}_{k}>2 .
$$

Then $\tilde{\mu}_{0,2}=0$, hence $\frac{\left|\tilde{\mu}_{0,2}\right|}{\tilde{\mu}_{1,1}}=0$.

One can give a statistical interpretation of our proposed shape elongation measure $\frac{\left|\tilde{\mu}_{0,2}\right|}{\tilde{\mu}_{1,1}}$. Indeed after renormalizing the total ink $\mu_{0,0}=\sum_{k=1}^{N} \rho\left(z_{k}\right)$ to one, one can view the pixel intensities as describing a discrete probability distribution. The standard deviation matrix of that distribution is then determined by the third row of the Pascal triangle, as stated in the following lemma:

Lemma 11. Consider the discrete image $I$ as a bivariate distribution with the joint probability mass function $P\left(X=x_{k}-x_{0}, Y=y_{k}-y_{0}\right)=\frac{\rho\left(z_{k}\right)}{\mu_{0,0}}, k=1,2, \ldots, N$. The covariance matrix $\Sigma$ of that distribution is given by

$$
\Sigma=\left(\begin{array}{cc}
\frac{\tilde{\mu}_{1,1}+\operatorname{Re}\left(\tilde{\mu}_{0,2}\right)}{2 \mu_{0,0}} & -\frac{\operatorname{Im}\left(\tilde{\mu}_{0,2}\right)}{2 \mu_{0,0}} \\
-\frac{\operatorname{Im}\left(\tilde{\mu}_{0,2}\right)}{2 \mu_{0,0}} & \frac{\tilde{\mu}_{1,1}-\operatorname{Re}\left(\tilde{\mu}_{0,2}\right)}{2 \mu_{0,0}}
\end{array}\right) .
$$

Proof. Observe that

$$
\begin{aligned}
\frac{\tilde{\mu}_{1,1}}{\mu_{0,0}} & =\sum_{k=1}^{N}\left(z_{k}-z_{0}\right)\left(\bar{z}_{k}-\bar{z}_{0}\right) \frac{\rho\left(z_{k}\right)}{\mu_{0,0}}=\sum_{k=1}^{N}\left(\left(x_{k}-x_{0}\right)^{2}+\left(y_{k}-y_{0}\right)^{2}\right) \frac{\rho\left(z_{k}\right)}{\mu_{0,0}}, \\
\frac{\tilde{\mu}_{0,2}}{\mu_{0,0}} & =\sum_{k=1}^{N}\left(\bar{z}_{k}-\bar{z}_{0}\right)^{2} \frac{\rho\left(z_{k}\right)}{\mu_{0,0}} \\
& =\sum_{k=1}^{N}\left(\left(x_{k}-x_{0}\right)^{2}-\left(y_{k}-y_{0}\right)^{2}\right) \frac{\rho\left(z_{k}\right)}{\mu_{0,0}}-2 i \sum_{k=1}^{N}\left(x_{k}-x_{0}\right)\left(y_{k}-y_{0}\right) \frac{\rho\left(z_{k}\right)}{\mu_{0,0}} .
\end{aligned}
$$


Write $\Sigma=\left(\begin{array}{cc}\sigma_{x}^{2} & \rho_{X Y} \sigma_{x} \sigma_{y} \\ \rho_{X Y} \sigma_{x} \sigma_{y} & \sigma_{y}^{2}\end{array}\right)$. Since the random variables $X, Y$ both have zero mean, we have

$$
\begin{aligned}
& \sigma_{x}^{2}=\sum_{k=1}^{N}\left(x_{k}-x_{0}\right)^{2} \frac{\rho\left(z_{k}\right)}{\mu_{0,0}}, \quad \sigma_{y}^{2}=\sum_{k=1}^{N}\left(y_{k}-y_{0}\right)^{2} \frac{\rho\left(z_{k}\right)}{\mu_{0,0}}, \\
& \rho_{X Y} \sigma_{x} \sigma_{y}=\sum_{k=1}^{N}\left(x_{k}-x_{0}\right)\left(y_{k}-y_{0}\right) \frac{\rho\left(z_{k}\right)}{\mu_{0,0}} .
\end{aligned}
$$

Then one can check that

$$
\frac{\tilde{\mu}_{1,1}}{2 \mu_{0,0}}+\frac{\operatorname{Re}\left(\tilde{\mu}_{0,2}\right)}{2 \mu_{0,0}}=\sigma_{x}^{2}, \quad-\frac{\operatorname{Im}\left(\tilde{\mu}_{0,2}\right)}{2 \mu_{0,0}}=\rho_{X Y} \sigma_{x} \sigma_{y}, \quad \frac{\tilde{\mu}_{1,1}}{2 \mu_{0,0}}-\frac{\operatorname{Re}\left(\tilde{\mu}_{0,2}\right)}{2 \mu_{0,0}}=\sigma_{y}^{2} .
$$

Recall that, in order to obtain the standard deviation $m_{2}(\theta)$ of the projection of a bivariate distribution onto the line with direction vector $(x, y)=r(\cos \theta, \sin \theta)$, one can simply project the standard deviation matrix $\Sigma$ onto $(x, y):\left(\begin{array}{ll}\cos \theta & \sin \theta\end{array}\right) \Sigma\left(\begin{array}{c}\cos \theta \\ \sin \theta\end{array}\right)=m_{2}(\theta)$.

It is easy to check that the relationship between the shape elongation $\frac{\left|\tilde{\mu}_{0,2}\right|}{\tilde{\mu}_{1,1}}$ and the eigenvalues $\lambda_{\max }, \lambda_{\min }$ of the standard deviation matrix $\Sigma$ is $\frac{\left|\tilde{\mu}_{0,2}\right|}{\tilde{\mu}_{1,1}}=\left|\frac{\lambda_{\max }-\lambda_{\min }}{\lambda_{\max }+\lambda_{\min }}\right|$.

\subsection{Rotational symmetry}

We have seen in the last section that an image $I$ having an $\tilde{N}$-FRS has $\tilde{\mu}_{0,2}=0$. More generally, we have the following lemmas, which was used in [5] as the basis for a HAZMAT sign recognition method.

Lemma 12. Let $\tilde{N}$ be a (finite) integer. If an image I has an $\tilde{N}$-fold rotation symmetry and if $\frac{l-j}{\tilde{N}}$ is not an integer, then $\tilde{\mu}_{j, l}=0$. Conversely, if $\tilde{\mu}_{j, l}=0$ for all $\frac{l-j}{\tilde{N}}$ that are not an integer, then the image $I$ has an $\tilde{N}$-fold rotation symmetry ${ }^{6}$.

Proof. $\Rightarrow$ Let us rotate $I$ clockwise around the origin by $\frac{2 \pi}{\tilde{N}}$. Due to its symmetry, the rotated image $I^{\prime}$ must be the same as the original one. In particular, it must hold

$$
\tilde{\mu}_{j, l}^{\prime}=e^{2 \pi i(l-j) / \tilde{N}} \tilde{\mu}_{j, l}=\tilde{\mu}_{j, l} .
$$

Since $\frac{l-j}{\tilde{N}}$ is not an integer, this equation can be fulfilled only if $\tilde{\mu}_{j, l}=0$.

$\Leftarrow$ Suppose $\tilde{\mu}_{j, 0}=0$ for any $\frac{j}{\tilde{N}}$ not an integer of some finite integer $\tilde{N}$. Let us rotate $I$ clockwise around the origin by $\frac{2 k \pi}{\tilde{N}}$ for each $k=1,2, \ldots, \tilde{N}-1$, then $\tilde{\mu}_{j, 0}^{\prime}=e^{-2 \pi i j k / \tilde{N}} \tilde{\mu}_{j, 0}$ for each $k$ and all $j=0,1, \ldots, N-1$. For $\frac{j k}{\tilde{N}} \in \mathbb{Z}$, it is easy to check that $e^{-2 \pi i j k / \tilde{N}}=1$, hence $\tilde{\mu}_{j, 0}^{\prime}=\tilde{\mu}_{j, 0}$. For $\frac{j k}{\tilde{N}} \notin \mathbb{Z}, \frac{j}{\tilde{N}}$ is not an integer either. Then $\tilde{\mu}_{j, 0}=0=\tilde{\mu}_{j, 0}^{\prime}$. In this way we have $\tilde{\mu}_{j, 0}^{\prime}=\tilde{\mu}_{j, 0}$ for all $j=0,1, \ldots, N-1$. Since in the proof of Lemma 1 , we showed that $\left\{\tilde{\mu}_{j, 0}\right\}_{j=0}^{N-1}$ uniquely determine $I$, then we can conclude that $I$ and $I^{\prime}$ are the same for any rotation with angle $\frac{2 k \pi}{\tilde{N}}, k=1,2, \ldots, \tilde{N}-1$. Therefore the image $I$ has an $\tilde{N}$-fold rotation symmetry.

Remark 4. As $N$ increases, more and more columns of the Pascal triangle $T^{r}(I)$ become zero, so that in the limit case, as $N \rightarrow \infty$, all entries $\mu_{j, l}$ with $j \neq l$ of $T^{r}(I)$, for any order $r$, vanish. This limit case corresponds to $\infty$-fold rotation symmetry (e.g. circles), which however does not occur among discrete images.

\footnotetext{
${ }^{6}$ An analogue for the "if" part of this lemma for the case of a continuous image can be found in [3].
} 


\subsection{Reflection symmetry}

Consider the reflection of an image about the line through the origin with direction vector $(\cos (\theta) \sin (\theta))^{T}, \theta \in\left(-\frac{\pi}{2}, \frac{\pi}{2}\right]$. The point $z_{k}=x_{k}+i y_{k}$ is mapped to $\underline{z}_{k}=\bar{z}_{k} e^{i 2 \theta}=\left(x_{k} \cos (2 \theta)+\right.$ $\left.y_{k} \sin (2 \theta)\right)+i\left(x_{k} \sin (2 \theta)-y_{k} \cos (2 \theta)\right)$ under the reflection with its pixel intensity $\rho\left(z_{k}\right)$ staying the same. Then the new complex moment is

$$
\begin{aligned}
\underline{\mu}_{j, l} & =\sum_{k=1}^{N} \underline{z}_{k}^{j} \underline{\bar{z}}_{k}^{l} \rho\left(\underline{z}_{k}\right)=\sum_{k=1}^{N}\left(\bar{z}_{k} e^{i 2 \theta}\right)^{j}\left(z_{k} e^{-i 2 \theta}\right)^{l} \rho\left(z_{k}\right) \\
& =\sum_{k=1}^{N} e^{i 2(j-l) \theta} \bar{z}_{k}^{j} z_{k}^{l} \rho\left(z_{k}\right)=e^{i 2(j-l) \theta} \sum_{k=1}^{N} \bar{z}_{k}^{j} z_{k}^{l} \rho\left(z_{k}\right)=\mu_{l, j} e^{i 2(j-l) \theta}, \quad \forall j, l \in \mathbb{Z}_{\geq 0} .
\end{aligned}
$$

Therefore the moment matrix for the new image $\underline{I}$ after reflection is

$$
\tau_{N}(\underline{I})=\left(\begin{array}{ccccc}
1 & 0 & 0 & \cdots & 0 \\
0 & e^{-i 2 \theta} & 0 & \cdots & 0 \\
0 & 0 & e^{-i 4 \theta} & \cdots & 0 \\
\vdots & \vdots & \vdots & \ddots & \vdots \\
0 & \cdots & \cdots & 0 & e^{-i 2(N-1) \theta}
\end{array}\right) \tau_{N}(I)^{T}\left(\begin{array}{ccccc}
1 & 0 & 0 & \cdots & 0 \\
0 & e^{i 2 \theta} & 0 & \cdots & 0 \\
0 & 0 & e^{i 4 \theta} & \cdots & 0 \\
\vdots & \vdots & \vdots & \ddots & \vdots \\
0 & \cdots & \cdots & 0 & e^{i 2(N-1) \theta}
\end{array}\right) .
$$

We can conclude from the above relation that if an image is symmetric with respect to the $x$-axis (i.e. $\theta=0$ ), then we will have $\tau_{N}(I)=\tau_{N}(I)^{T}$, i.e. $\mu_{j, l}=\mu_{l, j}$ for all $j, l \in \mathbb{Z}_{\geq 0}$. Since $\mu_{j, l}=\bar{\mu}_{l, j}$ by definition, this means that all the $\mu_{j, l}$ 's are real ${ }^{7}$.

Similarly, if an image is symmetric with respect to the $y$-axis (i.e. $\theta=\frac{\pi}{2}$ ), we can conclude that $\mu_{j, l}$ 's are real for $j, l$ of the same parity and $\mu_{j, l}$ 's are imaginary for $j, l$ of opposite parity.

More generally, we have the following result:

Lemma 13. A discrete image is symmetric with respect to reflections about a line through the origin with direction $\left(\begin{array}{cc}\cos \theta_{0} & \sin \theta_{0}\end{array}\right)^{T}$

$$
\Longleftrightarrow \quad \tan (l-j) \theta_{0}=-\frac{\operatorname{Im}\left(\mu_{j, l}\right)}{\operatorname{Re}\left(\mu_{j, l}\right)}, \quad j, l=0,1, \ldots
$$

Proof. Notice that with reflection symmetry, we have

$$
\begin{aligned}
m_{n}\left(\theta_{0}-\theta\right) & =\frac{1}{2^{n}} \sum_{k=1}^{N}\left(z_{k} e^{-i\left(\theta_{0}-\theta\right)}+\bar{z}_{k} e^{i\left(\theta_{0}-\theta\right)}\right)^{n} \rho\left(z_{k}\right) \\
& =\frac{1}{2^{n}} \sum_{k=1}^{N}\left(\bar{z}_{k} e^{i 2 \theta_{0}} e^{-i\left(\theta_{0}+\theta\right)}+z_{k} e^{-i 2 \theta_{0}} e^{i\left(\theta_{0}+\theta\right)}\right)^{n} \rho\left(z_{k}\right) \\
& =\frac{1}{2^{n}} \sum_{k=1}^{N}\left(z_{k}^{\prime} e^{-i\left(\theta_{0}+\theta\right)}+\bar{z}_{k}^{\prime} e^{i\left(\theta_{0}+\theta\right)}\right)^{n} \rho\left(\bar{z}_{k}^{\prime} e^{i 2 \theta_{0}}\right) \quad\left(\text { denote } z_{k}^{\prime}=\bar{z}_{k} e^{i 2 \theta_{0}}\right) \\
& =\frac{1}{2^{n}} \sum_{k=1}^{N}\left(z_{k}^{\prime} e^{-i\left(\theta_{0}+\theta\right)}+\bar{z}_{k}^{\prime} e^{i\left(\theta_{0}+\theta\right)}\right)^{n} \rho\left(z_{k}^{\prime}\right)=m_{n}\left(\theta_{0}+\theta\right) .
\end{aligned}
$$

Conversely, if $m_{n}\left(\theta_{0}-\theta\right)=m_{n}\left(\theta_{0}+\theta\right)$ for an image $I=\left\{\left(z_{k}, \rho\left(z_{k}\right)\right)\right\}_{k=1}^{N}$, then for its reflection image $I_{r}=\left\{\left(\bar{z}_{k} e^{i 2 \theta_{0}}, \rho\left(z_{k}\right)\right)\right\}_{k=1}^{N}$, there is $m_{n}^{r}\left(\theta_{0}+\theta\right)=m_{n}\left(\theta_{0}-\theta\right)=m_{n}\left(\theta_{0}+\theta\right)$ for

\footnotetext{
${ }^{7}$ For the continuous analogue, the fact that reflecting an object horizontally transforms complex moments into their conjugate was previously noted in [3].
} 
any $\theta \in(-\pi, \pi]$. From Lemmas 1 and 4 we can conclude that the image reconstructed from $\left\{m_{n}^{r}\left(\theta_{j}\right), j=1, \ldots, n+1\right\}_{n=0}^{N-1}$ and $\left\{m_{n}\left(\theta_{j}\right), j=1, \ldots, n+1\right\}_{n=0}^{N-1}$ will be the same, i.e. $I_{r}=I$. Hence the image has reflection symmetry.

By equation (5), we know

$$
\begin{aligned}
& m_{n}\left(\theta_{0}+\theta\right)=\frac{1}{2^{n}} \sum_{l=0}^{n}\left(\begin{array}{l}
n \\
l
\end{array}\right) \mu_{l, n-l} e^{i(n-2 l)\left(\theta_{0}+\theta\right)}, \\
& m_{n}\left(\theta_{0}-\theta\right)=\frac{1}{2^{n}} \sum_{l=0}^{n}\left(\begin{array}{c}
n \\
l
\end{array}\right) \mu_{l, n-l} e^{i(n-2 l)\left(\theta_{0}-\theta\right)} .
\end{aligned}
$$

Then $m_{n}\left(\theta_{0}+\theta\right)=m_{n}\left(\theta_{0}-\theta\right)$, i.e. $m_{n}\left(\theta_{0}+\theta\right)-m_{n}\left(\theta_{0}-\theta\right)=0$ can be written as

$$
\begin{aligned}
& \frac{1}{2^{n}} \sum_{l=0}^{n}\left(\begin{array}{l}
n \\
l
\end{array}\right) \mu_{l, n-l} e^{i(n-2 l) \theta_{0}}\left(e^{i(n-2 l) \theta}-e^{-i(n-2 l) \theta}\right)=0 \\
& \Leftrightarrow \quad \sum_{l=0}^{n}\left(\begin{array}{l}
n \\
l
\end{array}\right) \mu_{l, n-l} e^{i(n-2 l) \theta_{0}}(2 i \sin (n-2 l) \theta)=0 \\
& \Leftrightarrow \quad \sum_{l=0}^{n}\left(\begin{array}{l}
n \\
l
\end{array}\right) \mu_{l, n-l} e^{i(n-2 l) \theta_{0}} \sin (n-2 l) \theta=0 \\
& \Leftrightarrow \quad \sum_{l=0}^{\left\lfloor\frac{n-1}{2}\right\rfloor}\left(\begin{array}{l}
n \\
l
\end{array}\right) \sin (n-2 l) \theta\left(\mu_{l, n-l} e^{i(n-2 l) \theta_{0}}-\mu_{n-l, l} e^{i(2 l-n) \theta_{0}}\right)=0 \\
& \Leftrightarrow \quad \sum_{l=0}^{\left\lfloor\frac{n-1}{2}\right\rfloor}\left(\begin{array}{l}
n \\
l
\end{array}\right) \sin (n-2 l) \theta 2 i \operatorname{Im}\left(\mu_{l, n-l} e^{i(n-2 l) \theta_{0}}\right)=0 \\
& \Leftrightarrow \quad \sum_{l=0}^{\left\lfloor\frac{n-1}{2}\right\rfloor}\left(\begin{array}{l}
n \\
l
\end{array}\right) \sin (n-2 l) \theta\left(\operatorname{Re}\left(\mu_{l, n-l}\right) \sin (n-2 l) \theta_{0}+\operatorname{Im}\left(\mu_{l, n-l}\right) \cos (n-2 l) \theta_{0}\right)=0 .
\end{aligned}
$$

The above equation is true for any $\theta \in\left(-\frac{\pi}{2}, \frac{\pi}{2}\right]$, so it is true if and only if

$$
\operatorname{Re}\left(\mu_{l, n-l}\right) \sin (n-2 l) \theta_{0}+\operatorname{Im}\left(\mu_{l, n-l}\right) \cos (n-2 l) \theta_{0}=0, \quad l=0,1, \ldots,\left\lfloor\frac{n-1}{2}\right\rfloor,
$$

i.e.

$$
\tan (n-2 l) \theta_{0}=-\frac{\operatorname{Im}\left(\mu_{l, n-l}\right)}{\operatorname{Re}\left(\mu_{l, n-l}\right)}, \quad l=0,1, \ldots,\left\lfloor\frac{n-1}{2}\right\rfloor .
$$

Since $\mu_{n-l, l}=\bar{\mu}_{l, n-l},(16)$ can be written as

$$
\tan (l-j) \theta_{0}=-\frac{\operatorname{Im}\left(\mu_{j, l}\right)}{\operatorname{Re}\left(\mu_{j, l}\right)}, \quad \forall j=0,1, \ldots, \quad l=0,1, \ldots
$$

In this way, for a given discrete image, we can check (17) to decide whether it is symmetric with respect to a certain line or not. If it is, then the line is at an angle $\theta$ given by

$$
\theta= \begin{cases}\arctan \left(-\frac{\operatorname{Im}\left(\mu_{j, j+1}\right)}{\operatorname{Re}\left(\mu_{j, j+1}\right)}\right) & \text { if } \operatorname{Re}\left(\mu_{j, j+1}\right) \neq 0, \forall j=0,1, \ldots, \\ \frac{\pi}{2} & \text { otherwise. }\end{cases}
$$




\section{Experiments and results}

To illustrate the application of the Pascal triangle to symmetry detection, we used our results to design a simple reflection symmetry detection method $^{8}$. We tested this method on images from the MPEG-7 CE Shape-1 Part-B data set ${ }^{9}$. The data set includes 1400 binary images. The images are divided into 70 object classes, each object class containing 20 images. All our ground truth data and classification results can be downloaded from https://engineering. purdue.edu/ mboutin/symmetric_shapes.

\subsection{Horizontally symmetric object detection experiment}

In this experiment, we identified images that have a horizontal axis of reflection symmetry using only the first four rows of the Pascal triangle. Our data set consists of 320 shapes from the MPEG-7 shape database. Specifically, we included all 20 images contained in each of the following 16 classes: Bird, Device1-Device5, Device7-Device9, Watch, Cup, Dog, Flatfish, Glas, Hat, Tree.

We first manually divided the data into two sets. One set, called Set 1a, was assigned all objects that appeared to have a clear horizontal symmetry axis, up to some minor details. The remaining set, called Set 2a, was assigned the remaining images. Set 1a and Set 2a contain 113 and 207 images respectively. As one can see by inspecting Set 2a (see for example the images in Fig. 5(c) and (d)), our classification was quite strict. Indeed, we excluded many objects that could be declared symmetric under a greater tolerance for error. We thus created a second grouping allowing for more errors: Set $1 b$ and Set $2 b$, which are the data sets resulting from this more lenient definition of symmetry.

Recall that, by Lemma 13, an image has a horizontal axis of symmetry if and only if all the entries of its Pascal triangle are real. Since we are focusing on the symmetry of the object contained in the image, as opposed to the image itself, we need to consider the translation invariant Pascal triangle consisting of the centralized moments $\tilde{\mu}_{j, l}$. Thus horizontally symmetric objects should be recognizable by considering the magnitude of the imaginary part of each of its centralized moments. Note that $\tilde{\mu}_{0,0}$ and $\tilde{\mu}_{0,1}$ are always real and that $\tilde{\mu}_{2,0}=\overline{\tilde{\mu}}_{0,2}$. Thus, if we restrict ourselves to the first four rows of the Pascal triangle, for simplicity, then horizontally symmetric objects are characterized by the fact that $\tilde{\mu}_{0,2}, \tilde{\mu}_{0,3}$ and $\tilde{\mu}_{1,2}$ are real. In other words, objects that are approximately symmetric should have $\tilde{\mu}_{0,2}, \tilde{\mu}_{0,3}$ and $\tilde{\mu}_{1,2}$ with an imaginary part close to zero. In order to remove the scale ambiguity resulting from the arbitrary scale used to describe the pixel coordinates, we followed the approach described in Section 4, Example 2 to invariantize our coordinates with respect to scaling. Our specific classification criteria were:

$$
\begin{aligned}
& \text { if } \operatorname{Im}\left(\frac{\tilde{\mu}_{0,2}}{\tilde{\mu}_{1,1}}\right)^{2}+\operatorname{Im}\left(\frac{\tilde{\mu}_{0,3}}{\tilde{\mu}_{1,1}^{3 / 2}}\right)^{2}+\operatorname{Im}\left(\frac{\tilde{\mu}_{1,2}}{\tilde{\mu}_{1,1}^{3 / 2}}\right)^{2}<r^{2}, \\
& \text { then object is symmetric, } \\
& \text { else } \\
& \text { object is not symmetric, }
\end{aligned}
$$

where $r$ is a variable threshold.

In our experiments, we varied the threshold $r$ from 0.005 to 0.15 . For each value of $r$, we classified every image as either "symmetric" or not symmetric using the above mentioned

\footnotetext{
${ }^{8}$ The reader interested in the application of the Pascal triangle to the detection of rotational symmetries is invited to read [5].

${ }^{9}$ Shape data for the MPEG-7 core experiment CE-Shape-1, http://www.cis.temple.edu/ latecki/ TestData/mpeg7shapeB.tar.gz.
} 


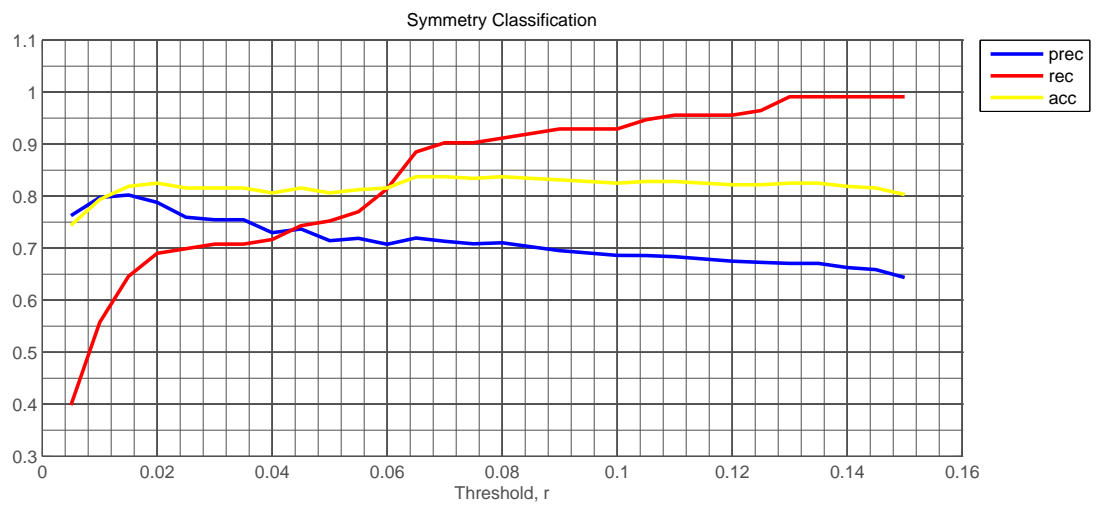

(a) Detection of horizontally symmetric objects using Set 1a and Set 2a data set and the first four rows of the Pascal triangle. The max accuracy is $83.75 \%$.

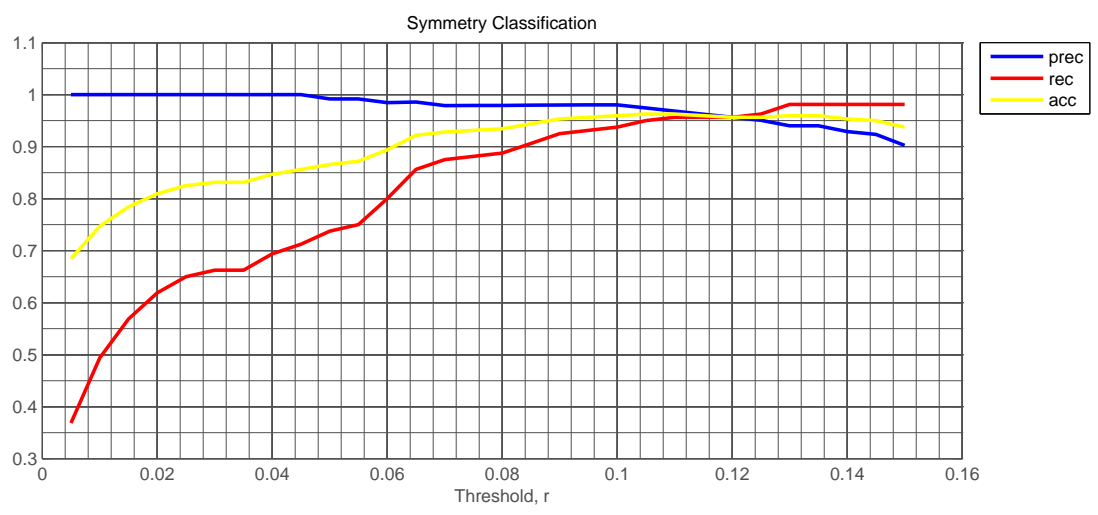

(b) Detection of horizontally symmetric objects using Set $1 \mathrm{~b}$ and Set $2 \mathrm{~b}$ data set and the first four rows of the Pascal triangle. The max accuracy is $96.25 \%$.

Figure 4. Precision, recall and accuracy for $r$ from 0.005 to 0.15 at increments of 0.005 .

criteria. We also computed the precision, recall, and accuracy for each value of $r$, where

$$
\begin{aligned}
& \text { precision }=\frac{\text { number of true positives }}{\text { number of true positives }+ \text { false positives }}, \\
& \text { recall }=\frac{\text { number of true positives }}{\text { number of true positives }+ \text { false negatives }}, \\
& \text { accuracy }=\frac{\text { number of true positives }+ \text { true negatives }}{\text { number of true positives }+ \text { true negatives }+ \text { false positives }+ \text { false negatives }} .
\end{aligned}
$$

The results obtained when using the data sets Set 1a and Set 2a are plotted in Fig. 4(a), and those obtained using Set $1 \mathrm{~b}$ and Set $2 \mathrm{~b}$ are plotted in Fig. 4(b). Observe that the maximum accuracy for the first data set, $83.75 \%$ (obtained around $r=0.07$ ), goes up to $96.25 \%$ (obtained around $r=0.11$ ) for the second data set. This is consistent with the fact that the second data set was constructed using a greater tolerance for error: after all, we are only using the first four rows of the triangle to classify the shape. Indeed, the shapes that were moved from Set $2 \mathrm{a}$ to Set $1 \mathrm{~b}$ caused the number of false positive to decrease and thus the precision to increase correspondingly. Fig. 5 illustrates some of our results.

\subsection{Symmetric objects (any axis) detection experiment}

In this experiment we identified images that have an axis of reflection symmetry. Our data set consists of 200 shapes from the following 10 classes of the MPEG-7 shape database: Beetle, Bell, Bird, Butterfly, Camel, Cattle, Classic, Crown, Horseshoe, Lizzard. 


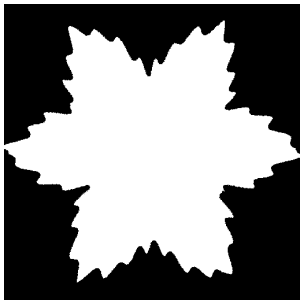

(a) Set 1a shapes classified as symmetric.
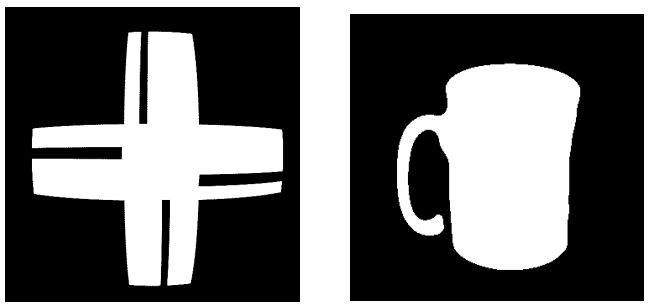

(c) Set 2a shapes (not in Set 1a) classified as symmetric.
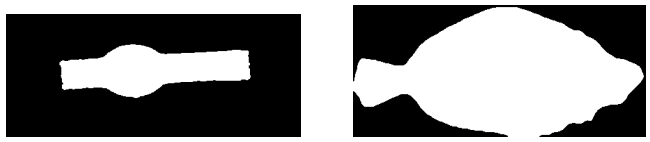

(b) Set 1a shapes classified as not symmetric.
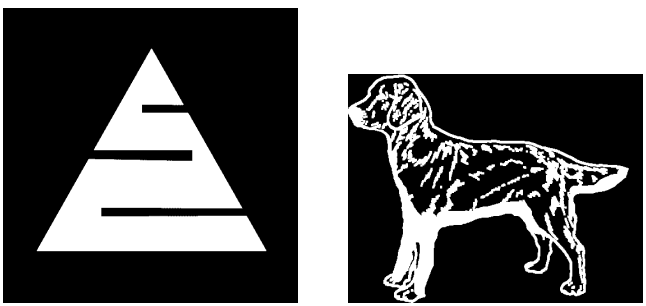

(d) Set 2a shapes (not in Set 1a) classified as not symmetric.

Figure 5. Small selection of shapes chosen from the MPEG-7 shape database, $r=0.07$.

We first manually divided the data set into two sets. One set, called Set 1, was assigned all objects in the classes of Beetle, Bell, Butterfly, Crown and Horseshoe. Because these objects all represent shapes that have a natural axis of symmetry. The remaining set, called Set 2, was assigned the images in the classes of Bird, Camel, Cattle, Classic and Lizzard. Set 1 and Set 2 each contains 100 images.

Recall that, by Lemma 13, an image is symmetric with respect to reflections about a line through the origin with direction $\left(\begin{array}{cc}\cos \theta_{0} & \sin \theta_{0}\end{array}\right)^{T}$ if and only if $\tan (l-j) \theta_{0}=-\frac{\operatorname{Im}\left(\mu_{j, l}\right)}{\operatorname{Re}\left(\mu_{j, l}\right)}$, for all $j, l \in \mathbb{Z}_{+}$. Since we are focusing on the symmetry of the object contained in the image, as opposed to the image itself, we need to consider the translation invariant Pascal triangle consisting of the centralized moments $\tilde{\mu}_{j, l}$. Thus the symmetry axis of symmetric objects should be recognizable by considering the arc tangent of the negative ratio of the imaginary part and the real part of each of its centralized moments. Note that the arc tangent of an angle are always between $-\frac{\pi}{2}$ and $\frac{\pi}{2}$, hence there will be some ambiguity when deciding $\theta_{0}$ from $\arctan \left(k \theta_{0}\right)$ with $|k|>1$. Also note that $\tilde{\mu}_{0,1}$ is always zero and $\tilde{\mu}_{j, l}=\overline{\tilde{\mu}}_{l, j}$. Thus for simplicity, we characterized the symmetry axis of symmetric objects by the fact that

$$
\theta_{0}=\arctan \left(-\frac{\operatorname{Im}\left(\tilde{\mu}_{1,2}\right)}{\operatorname{Re}\left(\tilde{\mu}_{1,2}\right)}\right)=\arctan \left(-\frac{\operatorname{Im}\left(\tilde{\mu}_{2,3}\right)}{\operatorname{Re}\left(\tilde{\mu}_{2,3}\right)}\right)=\arctan \left(-\frac{\operatorname{Im}\left(\tilde{\mu}_{3,4}\right)}{\operatorname{Re}\left(\tilde{\mu}_{3,4}\right)}\right) .
$$

In other words, objects that are approximately symmetric with respect to an axis of angle $\theta_{0}$ should have $\arctan \left(-\frac{\operatorname{Im}\left(\tilde{\mu}_{1,2}\right)}{\operatorname{Re}\left(\tilde{\mu}_{1,2}\right)}\right), \arctan \left(-\frac{\operatorname{Im}\left(\tilde{\mu}_{2,3}\right)}{\operatorname{Re}\left(\tilde{\mu}_{2,3}\right)}\right)$ and $\arctan \left(-\frac{\operatorname{Im}\left(\tilde{\mu}_{3,4}\right)}{\operatorname{Re}\left(\tilde{\mu}_{3,4}\right)}\right)$ close to each other. Since we consider the ratio of the imaginary part and real part of each moment, it is not necessary to remove the scale ambiguity resulting from the arbitrary scale used to describe the pixel coordinates in this experiment, as the quantities we consider are already invariant under scaling. Also note that, if the symmetry axis of an image is of angle $\theta_{0}=\frac{\pi}{2}$, then $\theta_{0}=-\frac{\pi}{2}$ also defines the same symmetry axis, although the arc tangent of the angles near these two values are quite different. Taking these into account, our specific classification criteria were:

$$
\text { if } \quad|| \theta_{1}\left|-\frac{\pi}{2}\right|<T, \quad|| \theta_{2}\left|-\frac{\pi}{2}\right|<T, \quad|| \theta_{3}\left|-\frac{\pi}{2}\right|<T,
$$

then object is symmetric vertically, 


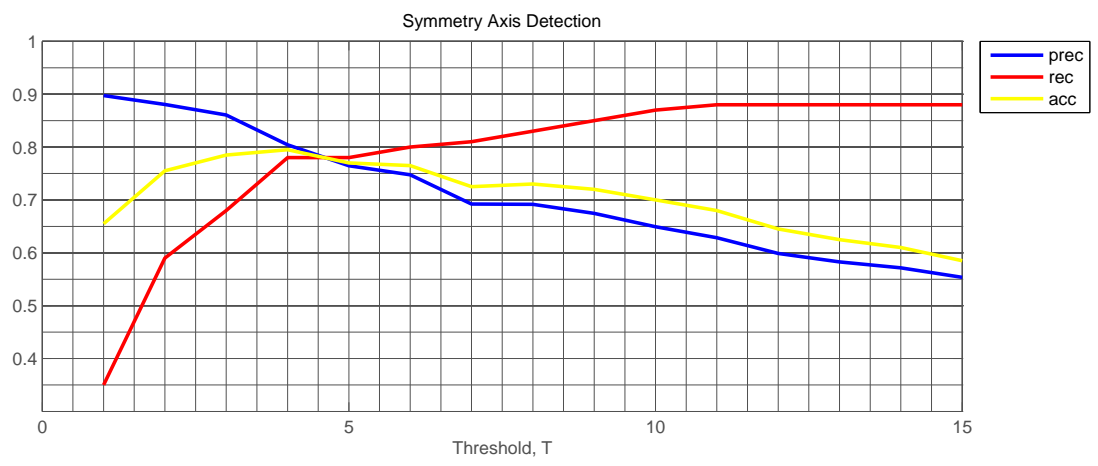

Figure 6. Symmetric objects detection using $\tilde{\mu}_{2,2}, \tilde{\mu}_{2,3}$ and $\tilde{\mu}_{3,4}$. The max accuracy is $79.5 \%$.

else if $\quad\left|\theta_{1}-\theta_{2}\right|<T, \quad\left|\theta_{2}-\theta_{3}\right|<T, \quad\left|\theta_{3}-\theta_{1}\right|<T$, then object is symmetric with symmetry axis $\theta_{0}=\frac{\theta_{1}+\theta_{2}+\theta_{3}}{3}$, else

object is not symmetric,

where $\theta_{1}=\arctan \left(-\frac{\operatorname{Im}\left(\tilde{\mu}_{1,2}\right)}{\operatorname{Re}\left(\tilde{\mu}_{1,2}\right)}\right), \theta_{2}=\arctan \left(-\frac{\operatorname{Im}\left(\tilde{\mu}_{2,3}\right)}{\operatorname{Re}\left(\tilde{\mu}_{2,3}\right)}\right), \theta_{3}=\arctan \left(-\frac{\operatorname{Im}\left(\tilde{\mu}_{3,4}\right)}{\operatorname{Re}\left(\tilde{\mu}_{3,4}\right)}\right)$ and $T$ is a variable threshold.

In our experiments, we varied the threshold $T$ from $1^{\circ}$ to $15^{\circ}$. For each value of $T$, we classified every image as either "symmetric" or not symmetric using the above mentioned criteria. And for each symmetric image, we found its symmetry axis. We also computed the precision, recall, and accuracy for each value of $T$.

The classification results obtained when using the data sets Set 1 and Set 2 are plotted in Fig. 6. Observe that the maximum accuracy for the data set is $79.5 \%$ at $T=4^{\circ}$. The accuracy of the experiment could be improved by using more moments: after all, we are only using three moments to classify the shapes. Indeed, using more moments would yield a more selective criterion, which should decrease the number of false positives and increase the number of true negatives. Fig. 7 illustrates some of our results.

\section{Conclusion and future work}

We have introduced the Pascal triangle of a discrete image, which is constructed using complexvalued moments. We obtained the relationship between the triangle and the Fourier series coefficients of the moment of the Radon transform of the image, that is, each row $n$ of the Pascal triangle contains the coefficients of the Fourier series of the $n$-th order moment of the Radon transform of the image. This relationship gives the moments a clear geometric interpretation. For example, $\frac{\mu_{0,3}}{8}$ of an image is the coefficient of $e^{i 3 \theta}$ in the third order moment $m_{3}(\theta)$ of the Radon transform of the image, and thus when $\left|\mu_{0,3}\right|$ is large, then the order three variation of the skewness of the projection is proportionally large.

We showed that the image can be fully reconstructed using a finite number of rows of the triangle. This fact, which is specific to discrete (finite) images, allows us to be able to derive necessary and sufficient conditions for the presence of various symmetries. It also allows us to conclude that the invariantized Pascal triangle separates the orbits of certain group actions. Indeed, by using the moving frame method we were able to invariantize the Pascal triangle with respect to translation, rotation and scaling, and by using the reconstruction property of the invariantized Pascal triangle, we were able to show the uniqueness of the reconstruction modulo these transformations. 

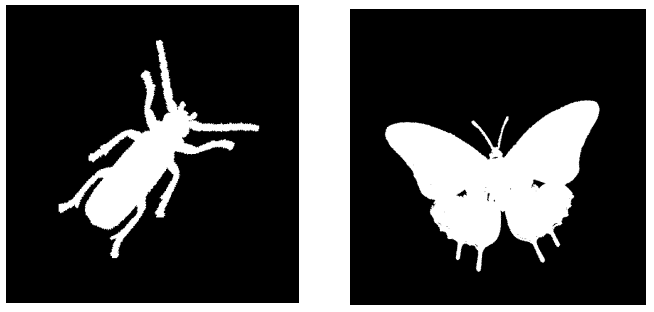

(a) Set 1 shapes classified as symmetric with axis $53.0^{\circ}$ and $94.5^{\circ}$ respectively.
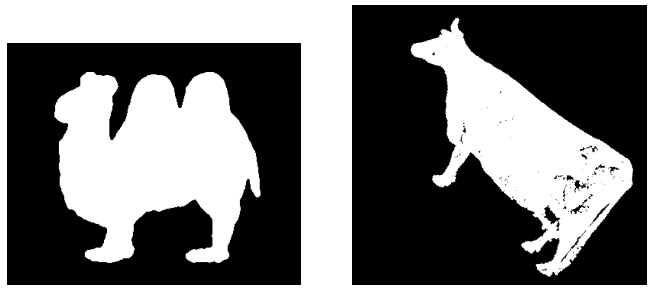

(c) Set 2 shapes classified as symmetric with axis $99.2^{\circ}$ and $136.1^{\circ}$ respectively.

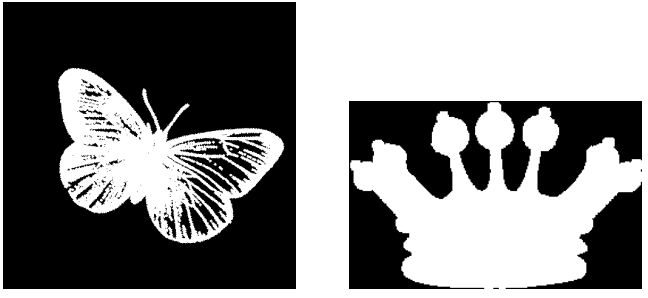

(b) Set 1 shapes classified as not symmetric.
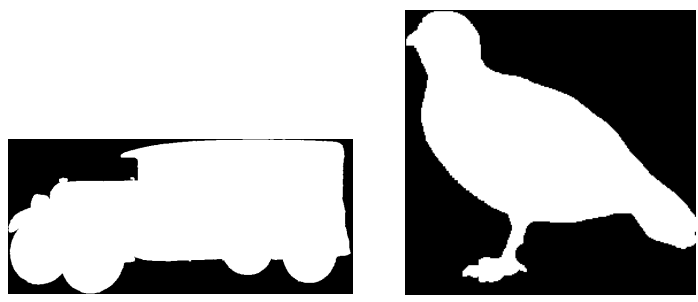

(d) Set 2 shapes classified as not symmetric.

Figure 7. Small selection of symmetric objects detection results for images in Set 1 and Set 2 with threshold $T=5$.

We tested the application of the Pascal triangle to the recognition of symmetric shapes from the MPEG-7 shape database. More specifically, we derived a simple method to detect horizontal symmetries using the first four rows of the triangle. We then tested this method using 16 object classes. We also derived a simple method to detect symmetry axes in objects using entries within only the first eight rows of the triangle. We then tested this method using 10 object classes.

Extension of our method to other group actions such as affine transforms should be doable using the moving frame method. Observe that our definition for $\mu_{j, l}$ naturally extends to vector valued pixel intensities. Therefore, it should be straightforward to extend our framework to the case of color images. We are looking forward to also extending this work to the case of 3D objects.

\section{Acknowledgments}

This research was supported in parts by NSF grant CCF-0728929.

\section{References}

[1] Fels M., Olver P.J., Moving coframes. I. A practical algorithm, Acta Appl. Math. 51 (1998), 161-213.

[2] Fels M., Olver P.J., Moving coframes. II. Regularization and theoretical foundations, Acta Appl. Math. 55 (1999), 127-208.

[3] Flusser J., Zitova B., Suk T., Moments and moment invariants in pattern recognition, John Wiley \& Sons Ltd., Chichester, 2009.

[4] Gustafsson B., He C., Milanfar P., Putinar M., Reconstructing planar domains from their moments, Inverse Problems 16 (2000), 1053-1070.

[5] Haddad A.W., Huang S., Boutin M., Delp E.J., Detection of symmetric shapes on a mobile device with applications to automatic sign interpretation, Proc. SPIE 8304 (2012), 83040G, 13 pages.

[6] Milanfar P., Verghese G.C., Karl W., Willsky A.S., Reconstruction polygons from moments with connections to array processing, IEEE Trans. Signal Process. 43 (1995), 432-443.

[7] Olver P.J., Classical invariant theory, London Mathematical Society Student Texts, Vol. 44, Cambridge University Press, Cambridge, 1999.

[8] Rostampour A.R., Madhvapathy P.R., Shape recognition using simple measures of projections, in Proceedings of Seventh Annual International Phoenix Conference on Computers and Communications (Scottsdale, AZ, 1988), IEEE, Arizona State University, 1988, 474-479. 